\title{
High-Resolution Topography and Albedo of the South Polar Layered Deposits on Mars
}

\author{
KEN E. HERKENHOFF ${ }^{1}$ AND BRUCE C. MURRAY
}

Division of Geological and Planetary Scienoes, Califormia Institute of Technology, Pasadena

\begin{abstract}
Using a new photoclinometric technique with high-resolution Mariner 9 images, maximum slopes of $10^{\circ}-20^{\circ}$ are found to occur on an exposure of layered deposits within the south polar residual cap of Mars. Stereophotogrammetry is used to constrain the photoclinometric solutions, which resolve layer thicknesses of $100-300 \mathrm{~m}$. Albedo variations are correlated with slope, indicating that frost is present on level areas. There is evidence for temporal changes in frost distribution in the 7 days ( $4^{\circ}$ of $L_{0}$ ) between the two images used in this study. The magnitude of the slopes derived here and consideration of the stability of water ice at the surface of the layered deposits strongly suggest the presence of a competent weathering rind. The weathered ourface may be composed of dark filamentary sublimation residue particles that protect the underlying ice from solar heating. This hypothesig is consistent with previous studies of the regional color and albedo of the layered deposits, which indicate that the deposits are slightly darker and less red than the bright dust that mantles much of the south polar region. Furthermore, the proposed weathering mechanism provides a plausible source of dark, saltating material for the Martian polar dune fields.
\end{abstract}

\section{INTRODUCTION}

The polar layered deposits on Mars are believed to record global climate cycles [Murray et al., 1972; Carr, 1982], but the details of the processes involved and their relative roles in layer formation remain obscure [Thomas et al., 1990]. The objective of this investigation is to derive improved knowledge of the topography and albedo of the southern layered deposits. Such results can constrain hypotheses for the formation and evolution of the deposits.

Previous efforts to derive slopes and heights of layered deposit exposures have been published [Drurisin and Blasius, 1975; Blasius et al., 1982; Howard et al., 1982a]. Blasius et al. [1982] found that layer contrasts were enhanced in the north polar deposits by retention of frost during the summer on relatively level slopes. By comparing such images with images of the same area taken during the spring (when the frost cover was essentially complete), they concluded that variations in layer composition also influence frost retention. However, the magnitude of albedo variations in the layered deposits has not been previously quantified due to limitations in photoclinometric techniques and the fundamental ambiguity between albedo and slope effects.

Murray et al. [1972] first recognized that most of the layering observed in the south polar region is due to the "staircase" topography of the deposits but noted that albedo variations between layers are indicated in some cases. Photoclinometry has not been applied successfully to the south polar layered deposits, however. Dzurisin and Blasius [1975] used stereophotogrammetry across exposures of layered deposits within the southern residual cap and found overall slopes in the range of $1^{\circ}-5^{\circ}$. Stereogrammetry can establish the ele-

\footnotetext{
${ }^{1}$ Now at Jet Propulsion Laboratory, California Institute of Technology, Pasadena

Copyright 1990 by the American Geophysical Union.

Paper number 90JB00163.

0148-0227/90/990JB-00163\$05.00
}

vation difference between distinct surface points but cannot resolve the topography of individual layers. Photoclinometric techniques can distinguish topographic features as small as the resolution of the image will permit but are sensitive to errors in estimates of level surface reflectance and atmospheric scattering [Howard et al., 1982a]. The analysis presented below combines stereophotogrammetry with twoimage photoclinometry [McEwen, 1985] to find the slopes and albedos of exposures of layered deposits in the south polar region of Mars. This technique makes use of two images of the same area taken with differing solar illumination, while previous photoclinometric profiling in the north polar layered deposits used only one image at a time and assumed a constant surface albedo and a Minnaert photometric function [Howard et al., 1982a]. Hapke's [1984] photometric function is used here and is recognized as the most physically realistic representation of surface reflectance [Veverka et al., 1986]. Hapke's function is not easily applied to photoclinometry [McEwen, submitted manuscript, 1990]. The method used here does not involve ratios of the photometric function, so that the surface roughness correction derived by Hapke [1984] may be included in the photoclinometric solution for the first time.

Details of the technique will be presented in the next section, after a description of the processing steps required to calibrate the images, to remove the effects of atmospheric dust scattering, and to produce smooth profiles. Photoclinometric solutions are very sensitive to the assumed values of atmospheric brightness [Howard et al., 1982a], so we have employed a multistream radiative transfer model to remove the effects of atmospheric dust scattering. Surface scattering parameters are chosen that produce overall topographic relief that is consistent with the stereogrammetric results described below. The results (discussed below) indicate that the majority of the albedo variations in exposures of the south polar layered deposits are due to the presence of frost, and that slopes of at least $10^{\circ}$ and perhaps $20^{\circ}$ occur. Such steep slopes have not been reported before and imply that the material comprising the layered deposits is rather 
competent. A weathering rind appears to have formed at the surface of the south polar layered deposits, protecting underlying water ice from solar heating.

\section{Data Processing and Modeling}

The highest-resolution images of the south polar region so far available were taken by the Mariner 9 orbiter in 1972. Hundreds of Mariner 9 B (narrow-angle) camera pictures were examined, and the best images of the layered deposits were selected. This subset of images was then searched for stereo coverage, with the additional requirement that the two solar azimuths in the stereo pair be as different as possible (the opposite of the usual requirement for stereo images). A large difference in solar illumination between two images of the same area permits two-image photoclinometry to be applied [McEwen, 1985]. Profiles that are nearly perpendicular to the solar azimuth in one image and more parallel in the other image are most desirable: in the former case the variations in reflectance along the profile are due mainly to albedo differences, while topographic modulation is more significant in the latter case. Finally, images that were acquired close in time are desirable because of changes in frost coverage in the polar regions.

Altogether, the above constraints are met by only a few Mariner 9 frames. The best single pair of Mariner 9 images of the south polar layered deposits that fulfills the above requirements is shown in Figures 1 and 2.

Throughout this paper they will be identified by their picture numbers, 173B1 and 188B2. The first three digits in each identifier represent the Mariner 9 orbit number (or "rev"), and the last digit is the frame number in the or- bital sequence. The images were taken about a week apart (February 8 and 15, 1972) by the narrow angle (B) camera during late southern summer $\left(L_{s}=342\right.$ and 346), when the polar cap had reached its minimum size. An earlier image, 124B7, was used to confirm the stereophotogrammetric results reported below.

Bright residual (and perhaps some seasonal) frost covers much of the area in the images and is interrupted by defrosted bands which have been previously shown to face toward the equator [Murray et al., 1972; Dzurisin and Blasius, 1975]. Layering is evident in these dark bands, especially in 188B2 (Figure 2). The images were not geometrically transformed to remove camera distortions or projected in any way that would have involved resampling the data. Such resampling of the images could have resulted in the loss or distortion of detail near the limit of resolution.

The direction of solar illumination is different in the two images, as shown in Figures 1 and 2. The image in which the illumination is nearly parallel to the strike of the layers will be referred to as the "albedo" image, because the variations in reflectance between layers is mainly due to variations in surface albedo. Similarly, the image in which the illumination is nearly perpendicular to the strike of the layers will be referred to as the "topography" image, because in this case variations in slope have a dominant effect on the reflectance.

\section{Radiometric Calibration}

The Mariner 9 images were corrected for noise and artifacts, and each pixel was converted into light intensity values using the procedures described by Herkenhoff et al. [1988]. Absolute calibration was achieved by comparing Mariner 9

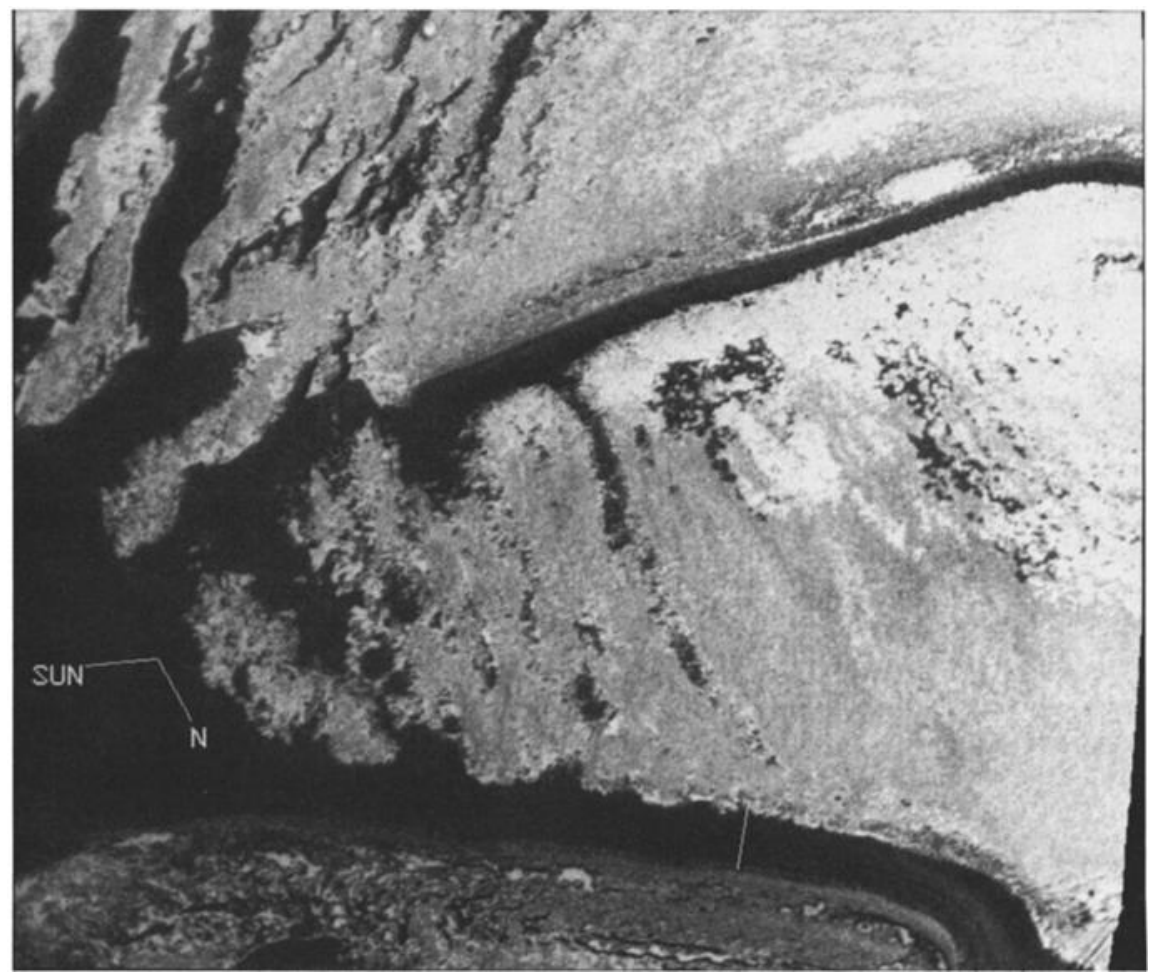

Fig. 1. Mariner 9 south polar residual cap image 173B1, corrected for atmospheric scattering. Profile 1 indicated by solid line across dark (unfrosted) band, with directions toward Sun and north pole indicated. Projected pixel size on the surface is $\sim 112 \mathrm{~m}$, so that features as small as $\sim 250 \mathrm{~m}$ across can be resolved. 


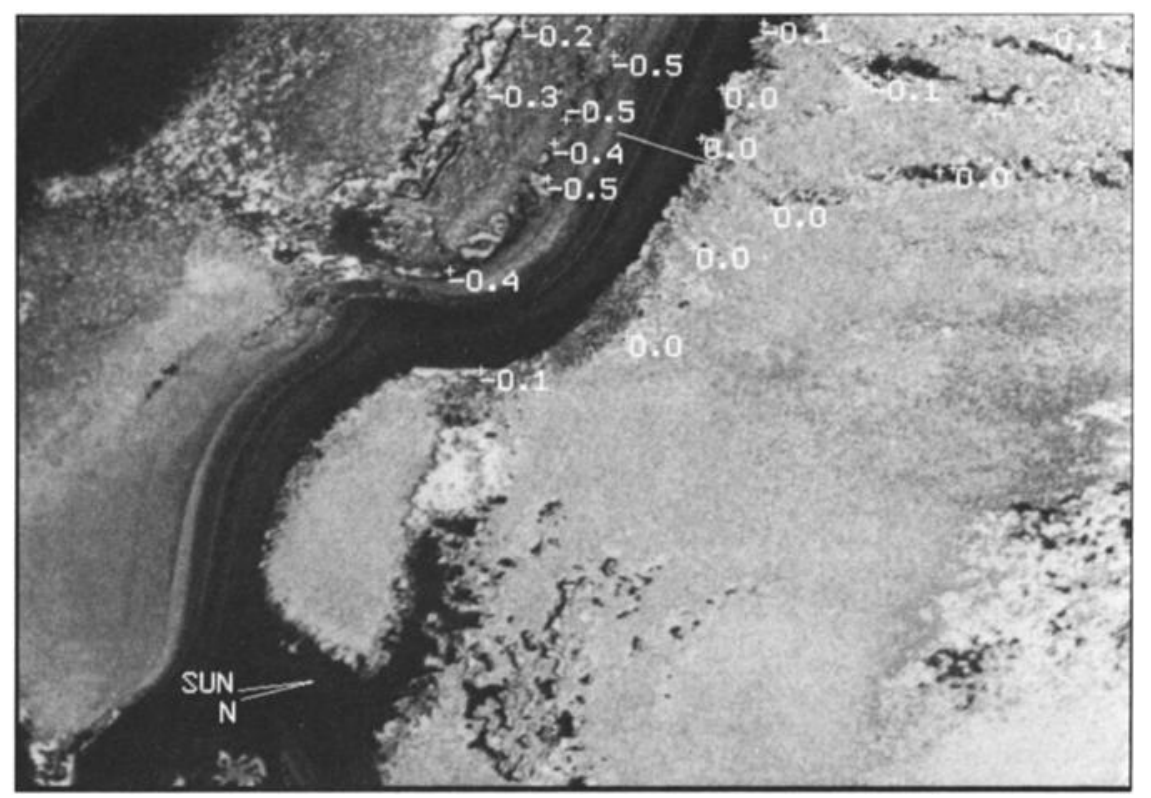

Fig. 2. Mariner 9 south polar residual cap image 188B2, corrected for atmospheric scattering. Profile 1 indicated by solid line across dark (unfrosted) band, with elevations of selected points relative to an arbitrary datum. Each cross symbol marks the location of a stereophotogrammetric elevation measurement. Projected pixel size on the surface is $\sim 96 \mathrm{~m}$, so that features as small as $\sim 200 \mathrm{~m}$ across can be resolved.

and Viking orbiter images of the same face of Phobos, assuming that the Viking calibration is correct. The absolute accuracy of the Mariner 9 calibration is therefore limited by the $13 \%(1 \sigma)$ absolute accuracy of the Viking orbiter cameras [Klaasen et al., 1977]. A full discussion of the absolute accuracy of spacecraft imaging is beyond the scope of this paper and will be the subject of a forthcoming publication, where it will be argued that a reasonable estimate of the $a b-$ solute accuracy of the Mariner 9 calibration by this method is about 20\% RMS. In any case, Mariner 9 absolute photometric uncertainty is no less than the $15 \%$ value estimated by Thorpe [1973] for the central $400 \times 400$ pixels at midscale. Relative errors within each image and between images are about $8 \%$ [Herkenhoff et al., 1988], and somewhat larger at low exposure levels. The effect of these uncertainties on our results will be discussed later.

\section{Stereophotogrammetry}

The camera pointing information for each image was refined using PICS software [Edwards, 1987; Batson, 1987] and a controlled digital mosaic of the south polar region provided by the U.S. Geological Survey. The errors in correcting the camera angles were never greater than 4 pixels, corresponding to a latitude offset of less than $0.01^{\circ}$. The improved pointing information was then used in an interactive PICS stereophotogrammetry program to yield the elevations plotted in Figure 2. The vertical offset in the intersections of vectors calculated by the program is $\mathbf{1 2 0} \mathrm{m}$ or less. This elevation uncertainty is comparable to the $122 \mathrm{~m}$ uncertainty estimated using equation (13) of Blasius [1973]. The overall topography and relief across the defrosted band (Figure 2) are in good agreement with the results presented by Dzurisin and Blagius [1975] and indicate that the traces of layers in the dark band are essentially horizontal. The strike of the layers is therefore assumed to be parallel to the trace seen in the images, so that the surface dips perpendicular to the trace.

Stereogrammetry was also used to find the elevations at the endpoints of two profiles (Table 1). The total relief of each profile (500 $\pm 90 \mathrm{~m}$ for profile $1 ; 300 \pm 160 \mathrm{~m}$ for profile 2) will be used to constrain photoclinometric solutions. Similar results are obtained from stereogrammetric analysis of another pair of images of the same area, 124B7 and $173 B 1$.

\section{Atmospheric Scattering Model}

In order to deduce the true magnitude of surface reflectance modulations arising from surface albedo or slope variations, the intensity contribution from atmospheric scattering must be estimated and removed. Atmospheric opacity is most directly estimated using measurements of brightness in shadows [Herkenhoff and Murray, 1990; McEwen, 1985]. No distinct shadows could be found in either of the images shown in Figures 1 and 2, so images taken on or near the same orbits were searched for shadows. Good shadows were found in high-resolution images of the south polar region taken during revs 173 and 187, but not rev 188. The images were radiometrically calibrated, then $5 \times 5$ pixel areas were averaged within shadows and on nearby level areas at the same incidence (solar zenith) angle. The dust scattering model described by Herkenhoff and Murray [1990] was used

TABLE 1. Latitudes and Longitudes of Profile Endpoints

\begin{tabular}{cccccc}
\hline & \multicolumn{2}{c}{ Start } & & \multicolumn{2}{c}{ End } \\
\cline { 2 - 3 } \cline { 5 - 6 } Profile & Latitude & Longitude & & Latitude & Longitude \\
\hline 1 & -86.91 & $\mathbf{3 4 6 . 8 4}$ & & -87.00 & 348.38 \\
2 & $\mathbf{- 8 6 . 6 2}$ & $\mathbf{3 5 0 . 1 5}$ & & $\mathbf{- 8 6 . 5 8}$ & $\mathbf{3 5 2 . 1 8}$ \\
3 & $\mathbf{- 8 7 . 0 1}$ & $\mathbf{3 4 5 . 1 2}$ & & $\mathbf{- 8 7 . 0 6}$ & $\mathbf{3 4 6 . 2 3}$ \\
\hline
\end{tabular}


to fit these data as shown in Figure 3. The single-scattering albedo of 0.81 , Henyey-Greenstein asymmetry parameter of 0.48 and extinction efficiency of 2.72 used in the model were interpolated to the Mariner $9 \mathrm{~B}$ camera effective wavelength of $0.56 \mu$ from the results of models of Viking orbiter color data [Herkenhoff and Murray, 1990].

The assumption of Lambertian surface scattering in the atmospheric model is clearly not valid in general for the surface of Mars. However, we feel that this approximation is sufficient within a relatively confined region because the direct surface reflectance is generally a small fraction of the total observed flux at high incidence angles (Figure 3). Deviations from Lambertian behavior are most significant in the direct surface reflection, and are less important in the calculation of diffuse reflection. The total reflectance at large incidence angles (upper points in Figure 3) is sensitive to changes in surface albedo, but insensitive to small changes in optical depth. The Lambert albedos (normal reflectances) in Figure 3 were therefore found by fitting the total reflectance measurements, then the optical depth was varied to fit the shadow data. Although the dust opacity over the south polar region was gradually decreasing during the Mariner 9 primary mission [Pang and Hord, 1973], a constant opacity of 0.30 over this short time interval is plausible.

The dust concentration in the atmospheric model decays exponentially with a scale height of $10 \mathrm{~km}$ up to a maximum of $50 \mathrm{~km}$, so that much of the total opacity is due to dust near the surface. Regional elevation differences will therefore affect the atmospheric opacity calculated by the model. The topography of south polar region is unknown except in the area of the residual cap [Dzurisin and Blasius, 1975]. The areas modeled in Figure 3 are several hundred kilometers from the residual cap, near the edge of the layered deposits. The radio occultation and stereogrammetric data presented by Dzurisin and Blasius [1975] indicate that the portion of the residual cap in Figures 1 and 2 is about 2 $\mathbf{k m}$ higher than the areas modeled for atmospheric scattering. The vertical dust distribution was therefore modified to account for the greater surface elevation in images 173B1 and $188 \mathrm{~B} 2$ by removing the bottom $2 \mathrm{~km}$ of dust from the model used to fit the shadow data. This effectively reduces the dust optical depth to $\mathbf{0 . 2 5}$.

In order to correct 173B1 and 188B2 for atmospheric effects, an average Lambert surface albedo must be chosen for use in the model. The width of the dark band of exposed layered terrain is less than an atmospheric scale height, so surface reflection from bright frost will be a significant component of the illumination of suspended dust particles. However, a surface albedo similar to those in Figure 3 is more appropriate for calculating the surface reflection of diffuse radiation from the atmosphere. We therefore expect that a surface albedo greater than that of the dark ground and less than that of the bright frost will most accurately correct for atmospheric effects in the dark band. The Lambert albedo of the residual frost at the effective wavelength of the B camera is near 0.5 [Herkenhoff and Murray, 1990], so we estimate that the proper surface albedo for atmospheric correction is between 0.3 and 0.5 in this case. Surface albedos greater than 0.45 resulted in excessive subtraction of atmospheric scattering and surface reflectances of zero in some areas. Lambert albedos between 0.3 and 0.45 are therefore indicated for the atmospheric model. The method by which we chose a surface albedo of 0.37 to correct for atmospheric effects will be described below.

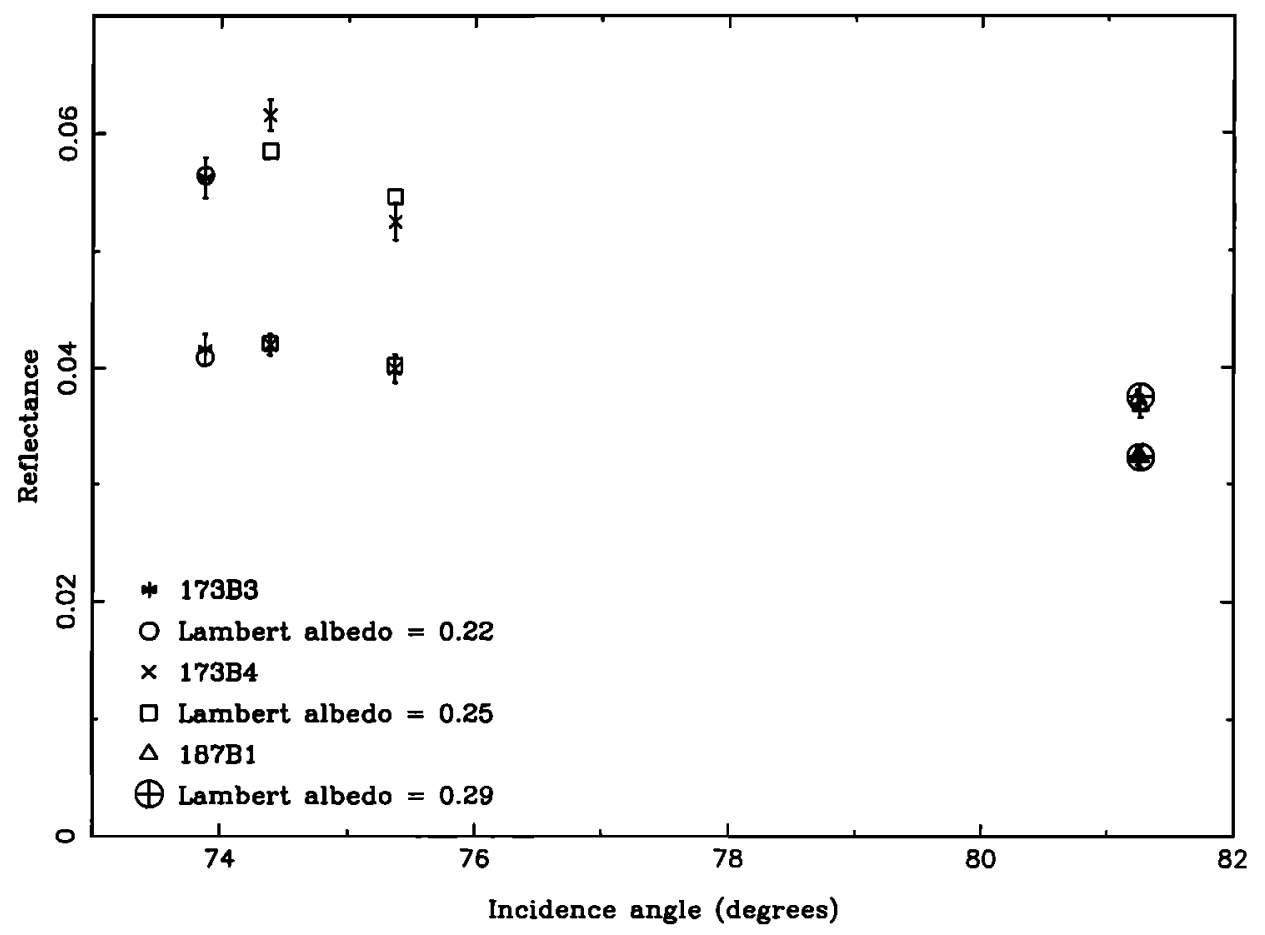

Fig. 3. Shadow (lower points) and nearby level surface (upper points) reflectance data. Error bars represent the standard deviation of values within $5 \times 5$ pixel areas. Model fits for optical depth $=0.30$ are shown. 


\section{Profiles}

Profile endpoints were located using surface features that were visible in both images (173B1 and 188B2). The latitudes and longitudes of the endpoints in the two images (Table 1) were derived using the stereogrammetric software described above. Incidence, emission, and phase angles at each of the profile endpoints were then found relative to a mean ellipsoidal surface using the corrected camera pointing information (Table 2). The errors in these angles are of the same order as the uncertainty in location of surface points, less than $0.01^{\circ}$.

It was necessary to include pixels adjacent to the line connecting profile endpoints because of noise in the images because the layers are barely resolved. Pixel values within a given range of the profile center line were projected perpendicularly to the center line, under the assumption that layers are perpendicular to the profile. In some cases the profile was not exactly perpendicular to the strike of the layers, so that only pixels close to the profile could be projected. In Figure 1, profile 1 is perpendicular to the layers, but in Figure 2 the same profile appears inclined due to the oblique viewing geometry. The best estimates of surface reflectance, shown in Figure 4, were obtained with a 6 or 8 pixel range (3-4 pixels on either side of the center line). A few points in some of the profiles were clearly erroneous (usually bit errors) and were deleted. The edited data were then averaged in 2-pixel bins along the profile length, as plotted in Figure 4. Smaller bins were tested, but resulted in much rougher profiles and are not realistic given the modulation transfer function of the camera [Cutts, 1974]. Two-pixel averages of data corrected for atmospheric effects were used in the photoclinometric modeling described below.

\section{Photometric Function}

In order to derive slopes from the reflectance profiles, the scattering properties of the surface are modeled using Hapke's [1984] photometric function. Hapke's function is sufficiently complicated that it will not be reproduced here. Certain parameters in the function do not vary with position along the profiles and were set in advance of any modeling. The phase angles of the Mariner 9 observations studied here are large enough that we neglect the backscatter function $B$ and set the backscatter parameter $h=0$.

Helfenstein [1988] noted that the Hapke function is inaccurate at high incidence angles for rough (mean macroscopic surface slope angle $\bar{\theta}>10^{\circ}$ ) surfaces, so we use $\bar{\theta}=5^{\circ}$. The layered deposits appear smooth down to the limit of resolution in Viking and Mariner 9 images, so this choice of $\bar{\theta}$ is plausible. Using $\bar{\theta}=0^{\circ}$ gives essentially the same numerical results as $\bar{\theta}=5^{\circ}$. For comparison, Arvidson et al. [1989] found that $\bar{\theta} \approx 6^{\circ}$ in dust deposits at the Viking Lander 1 site. Multiple scattering between macroscopic facets on a rough surface is not considered in Hapke's function [Buratti and Veverka, 1985] but should not be significant in this case.

Thorpe [1982] fit Hapke's [1981] $\left(\bar{\theta}=0^{\circ}\right)$ photometric function to Viking orbiter observations of Mars at small phase angles through both red and violet filters. The parameters he reports provide reasonable limits for the singlescattering albedo $w$ and phase coefficient $b$ of particles on the surface of the layered deposits. Color and albedo mapping by Herkenhoff and Murray [1990] indicates that the surface of the layered deposits in 173B1 and 188B2 is mantled by bright, red dust. Assuming that this dust has roughly the same optical properties as the bright dust in Arabia or Chryse studied by Thorpe [1982] and Arvidson et al. [1989], we should expect that $0.5 \leq w \leq 0.8$ at the effective wavelength of the $B$ camera. In any case, the single-scattering albedo of (nonvolatile) surface particles probably does not exceed 0.81 , the single-scattering albedo of the atmospheric dust particles.

The range of phase angles $\alpha$ in the images considered here is small enough (Table 2) that the simple phase function $P(\alpha)=1+b \cos (\alpha)$ is adequate. Thorpe [1982] fit lowphase observations using the same function and gives values of $b$ for various areas in both red and violet light. His results indicate that $0.2 \leq b \leq 0.5$ for bright areas at the effective wavelength of the $B$ camera.

The minimum reflectances in all of the profiles within each image are roughly equal, suggesting that they represent level areas. Assuming this to be the case, the minimum values can be modeled using the parameters described above and the geometric information in Table 2. The values of $w$ and $b$ were varied within reasonable limits to fit the minimum reflectances in both images simultaneously, assuming in addition that the albedo of the surface did not change in the week between the two images. A good fit to the data could be made after correcting for atmospheric effects using surface Lambert albedos between 0.35 and 0.4. A surface albedo of 0.37 yields reflectances that are well fit by $b=0.5$ and $w=0.75$. Next, it will be shown how these values were chosen to produce topographic profiles that are in agreement with stereogrammetric data.

\section{Photoclinometry}

A FORTRAN program was developed that iteratively finds the slope of a surface element required to match (within $1 \%$ of the standard deviations shown in Figure 4) each averaged reflectance datum using Hapke's photometric function.

TABLE 2. Incidence, Emission, and Phase Angles at Profile Endpoints

\begin{tabular}{|c|c|c|c|c|c|c|c|}
\hline \multirow[b]{2}{*}{ Image } & \multirow[b]{2}{*}{ Profile } & \multicolumn{3}{|c|}{ Start } & \multicolumn{3}{|c|}{ End } \\
\hline & & $\iota$ & $\epsilon$ & $\alpha$ & $\iota$ & $\epsilon$ & $\alpha$ \\
\hline 173B1 & 1 & 83.53 & 45.88 & 68.76 & 83.57 & 46.07 & 68.75 \\
\hline $173 B 1$ & 2 & $\mathbf{8 3 . 8 1}$ & 45.77 & 68.51 & 83.94 & 44.88 & 68.41 \\
\hline 173B1 & 3 & 83.41 & 45.89 & 68.87 & $\mathbf{8 3 . 4 4}$ & 46.01 & 68.85 \\
\hline 188B2 & 1 & 80.95 & 46.12 & 61.20 & 81.04 & 46.10 & 61.10 \\
\hline 188B2 & 2 & 80.67 & 45.59 & 61.37 & 80.65 & 45.39 & 61.36 \\
\hline $188 \mathrm{B2}$ & 3 & 81.04 & 46.34 & 61.15 & 81.09 & 46.31 & 61.09 \\
\hline
\end{tabular}



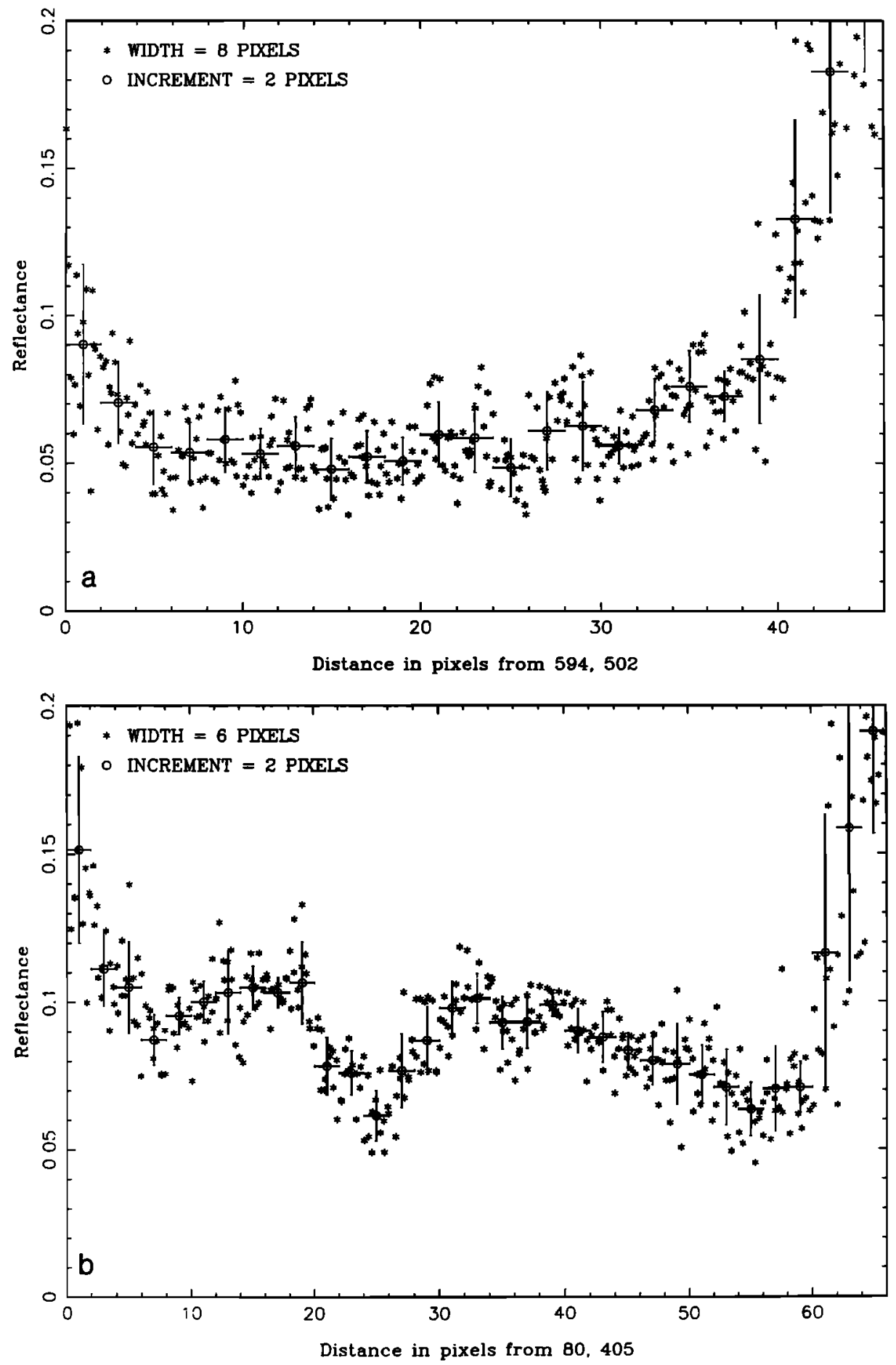

Fig. 4. Profile 1 data with averages of 2-pixel increments. Vertical error bars indicate standard deviation of pixel values in each increment. Increased reflectance near profile endpoints is due to inclusion of residual frost. (a) 173B1 ("albedo" image) data from 8-pixel wide swath around profile. (b) 188B2 ("topography" image) data from 6-pixel wide swath around profile.

The incidence, emission and phase angles at each point in the profile are interpolated from the angles at the profile endpoints (Table 2). The surface element is rotated about the strike of the layers (see Figure 5) until the reflectance is fit to within the specified tolerance. The incidence $\iota^{\prime}$ and emission $\epsilon^{\prime}$ angles on the rotated element are related to the interpolated incidence $\iota$ and emission $\epsilon$ angles of a level surface at the same location by

$$
\begin{aligned}
& \cos \iota^{\prime}=\cos \iota \cos \delta+\sin \iota \sin \delta \sin \phi \\
& \cos \epsilon^{\prime}=\cos \epsilon \cos \delta+\sin \epsilon \sin \delta \sin \psi
\end{aligned}
$$

where $\delta$ is the dip angle and $\phi$ and $\psi$ are the angles between the strike of the layers and the azimuths of the sun and spacecraft, respectively:

$$
\begin{aligned}
& \cos \phi=\frac{\cos \iota_{1}-\cos \iota_{2} \cos \gamma}{\sin \iota_{2} \sin \gamma} \\
& \cos \psi=\frac{\cos \epsilon_{1}-\cos \epsilon_{2} \cos \gamma}{\sin \epsilon_{2} \sin \gamma}
\end{aligned}
$$

Here the subscripts refer to the starting and ending points 


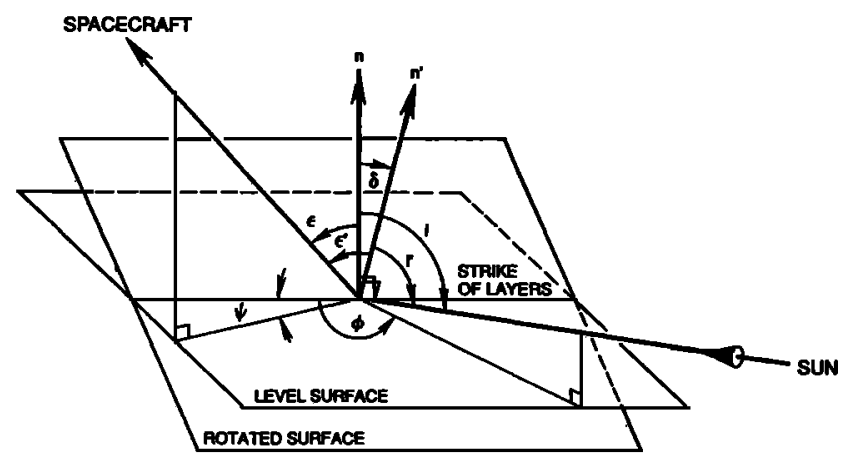

Fig. 5. Diagram illustrating geometric relationships defined in the text. Level surface normal denoted $n$, rotated surface normal denoted $n^{\prime}$.

of the profile, and $\gamma$ is the planetocentric angular difference between the endpoints of the profile, defined by

$$
\cos \gamma=\sin \lambda_{1} \sin \lambda_{2}+\cos \lambda_{1} \cos \lambda_{2} \cos \left(\beta_{1}-\beta_{2}\right)
$$

where $\lambda$ is latitude and $\beta$ is longitude of the endpoints, with the subscripts having the same meaning as above. The interpolated phase angle does not change as the surface element is rotated. The relationships between these angles are illustrated in Figure 5 and are similar to those presented by Howard et al. [1982a], with minor corrections. No correction is made for parallax offsets [Davis and Soderblom, 1984], as they should only be significant in profile 2 (discussed below).

It is first assumed that the single-scattering albedo of the surface particles is constant along the profiles, i.e., the vari- ations in reflectance along the profiles are due mainly to slopes. This is clearly not the case near the ends of the profiles where polar frost is included (see Figure 4a, where the solar illumination is nearly parallel to the strike), and erroneous results are expected in these areas. By ignoring the slopes derived near the ends of the profiles where frost is present, the overall relief of the profiles found by photoclinometry can be compared with the relief found using stereogrammetry (Figure 6). Such a comparison for profile 1 in 188B2 indicates that $w=0.75 \pm 0.02$ and $b=0.5 \pm 0.1$, as other values yield either more or less than $500 \pm 90 \mathrm{~m}$ of total relief. The single-scattering albedo $w$ and phase coefficient $b$ used in this model are consistent with the results of Thorpe [1982] and are applied to the other profiles in this study.

The sensitivity of the photoclinometric results to the type of photometric function assumed was tested by evaluating the topography along profile 1 using a Lambert function: $r=r_{0}(\alpha) \cos \iota$, where $r$ is the observed reflectance and $r_{0}(\alpha)$ is the reflectance at $\iota=0$ and phase angle $\alpha$. Because the phase angles in the two images are similar, ro was not allowed to vary between images. This simple photometric function with $r_{0}=0.12$ yields the same topography along profile 1 as the Hapke model (within the stereogrammetric uncertainty; see Figure 6). This comparison shows that the choice of photometric function does not significantly affect the photoclinometric results reported below.

Figure 7 shows that the topographic profile derived from the reflectance variations in $188 \mathrm{~B} 2$ is consistent with the 173B1 reflectance data within the standard deviations of the pixel values. The solar illumination in 173B1 is nearly parallel with the strike of the layers, so that surface albedo variations should dominate topographic modulation of the reflectance. The good agreement between the reflectance

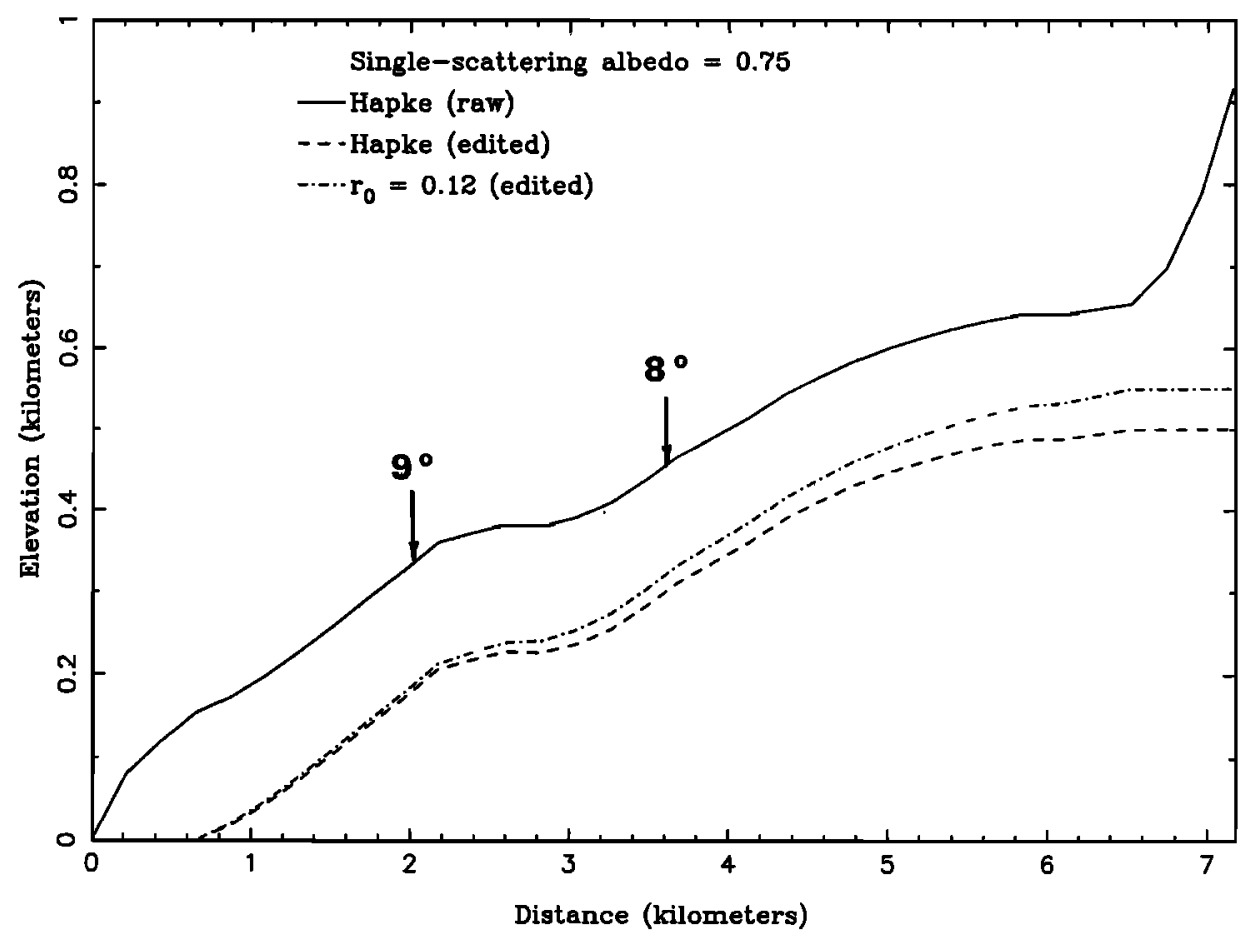

Fig. 6. Constant $w$ Hapke model topography for profile 1 with $b=0.5$. Dashed curve shows same profile with slopes set to zero in areas containing abundant frost. Dotted and dashed curve shows topography derived using Lambert photometric function with $r_{0}=\mathbf{0 . 1 2}$, also edited to remove erroneous slopes in frosted areas. Vertical exaggeration 5.2X; maximum slopes indicated on top curve are identical in other curves. 


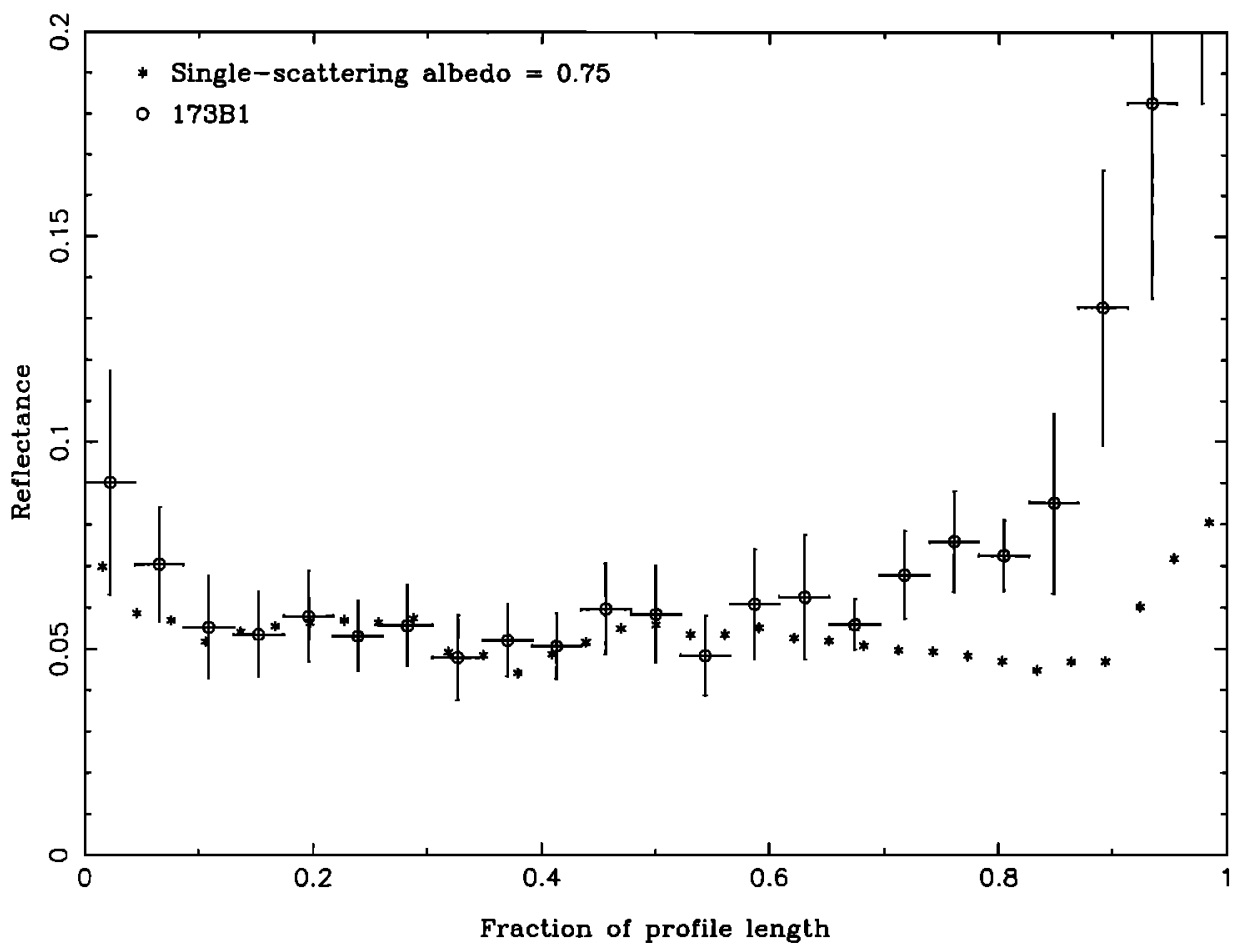

Fig. 7. Comparison of reflectance predicted by model topography with profile 1 data from "albedo" image.

predicted by the model and the data (except near the endpoints) indicates that the assumption of constant singlescattering albedo is valid within the uncertainties in the data and that temporal variations in frost cover are not significant along this profile. This result will be discussed further in the next section.

Alternatively, $w$ may be allowed to vary with distance along the profile. In this case, an initial single-scattering albedo is used to estimate the slope at each point in the topography image (188B2) as above except that the tolerance for model fitting is relaxed to $50 \%$ of the standard deviation of the reflectance data. The initial slopes are then interpolated to the points in the albedo image (173B1) and used evaluate the model reflectance at each point. If the model reflectance does not agree with the actual reflectance within $50 \%$ of the standard deviation of the data, the single-scattering albedo is iteratively perturbed until the reflectances converge. These albedos are then used to revise the slopes at each point in the topography image as above by interpolating $w$ at each point. The topographic profile found in this manner is then used to recalculate $w$ in the albedo image. The entire sequence is repeated until the solutions converge within the $0.5 \sigma$ tolerance, typically within three iterations. In the discussion that follows, this model will be referred to as the "general" model.

The resulting profile (Figure 8 ) has the same overall relief as the constant $w$ profile within the stereogrammetric uncertainty. Hence slopes of up to $9^{\circ}$ along this profile are consistent with the stereogrammetric data. Other combinations of Lambert surface albedo (in the atmospheric model), $w$ and $b$ can satisfy the stereogrammetric constraint only in the constant $w$ case, indicating that the parameters used here are most consistent with the data. These parameters were then used to model the slopes and albedo along two other profiles in this image pair (Figures 9-17).

\section{Results AND Discussion}

A limited amount of regional topographic information is revealed by stereophotogrammetric analysis of the image pair shown in Figures 1 and 2. The elevation data in Figure 2 indicate that the areas covered by the residual frost cap are either level or slope gently toward the south pole. Such a poleward slope may be responsible for the offset of the residual cap from the geometric pole, as suggested by Dzurisin and Blasius [1975]. The maximum slopes of $2.5^{\circ} \pm 1.5^{\circ} \mathrm{oc}-$ cur in the upper leftmost area of stereo coverage in Figure 2. Similar poleward facing slopes were found in the north polar layered deposits by Blasius et al. [1982].

\section{Profile 1}

Comparison of the profiles in Figure 4 indicates that there is some correlation between features in the two images. The prominent dip in reflectance $\sim 25$ pixels from the start of the profile in 188B2 is much more subdued in 173B1, while the smaller dip $\sim 36$ pixels from the starting point in $188 \mathrm{~B} 2$ is quite obvious 25 pixels from the starting point in 173B1. Because the layers in 173B1 are illuminated nearly parallel to their strike, we expect that topographic variations will have a small effect on the reflectance compared to albedo variations. This suggests that at least some of the reflectance differences in 173B1 are due to albedo variations.

However, the profile 1 data are consistent with a constant single-scattering albedo of 0.75 , as shown in Figures 6 and 7. The discrepancy between the model and the data in the last (far right) 0.3 of the profile in Figure 7 is probably due to the presence of seasonal frost during rev 173 that sublimed away by rev 188 . This hypothesis was tested by setting the 173B1 reflectance data in this area to the value at 0.67 of the profile length and recalculating the slopes 

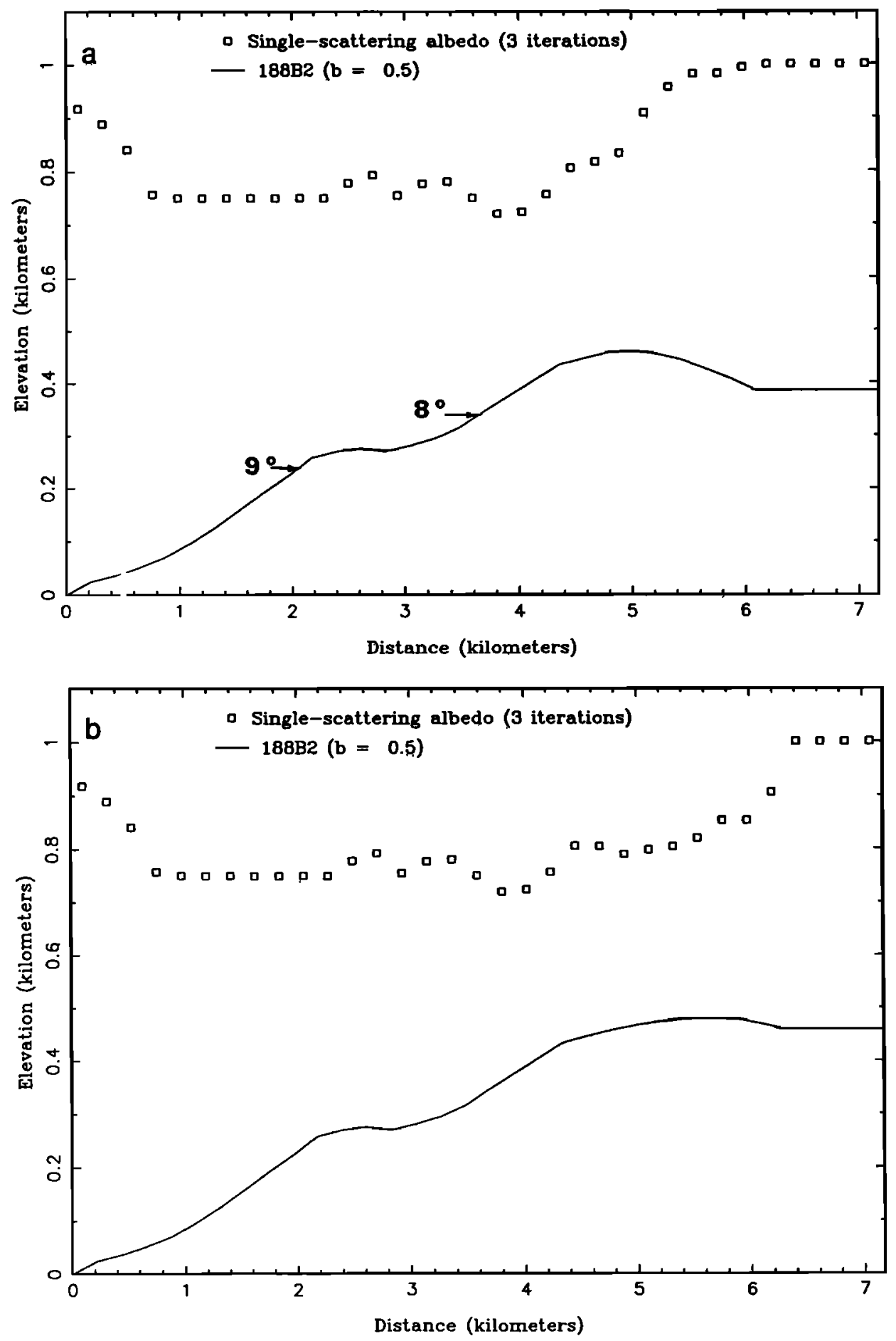

Fig. 8. Profile 1 topography and albedo from iterative model. Elevation and single-scattering albedo are plotted on the same vertical scale. Albedos of 1.0 in bright areas due to use of atmospheric model appropriate only for dark surface, not included in solution. Vertical exaggeration 4.7 $\times$; maximum slopes indicated. (a) Negative slope 5-6 $\mathrm{km}$ from starting point due to change in albedo between images. (b) Results for modified "albedo" image data, as described in the text.

using the general model. As shown in Figure 8, the raw data predict a negative (poleward) slope in this area, while the edited data yield a more realistic (nearly zero) slope. This illustrates a danger in assuming temporally invariant surface albedos in two-image photoclinometry in the polar regions. The analysis of profile 2, discussed below, further clarifies this problem.
In any case, the results shown in Figure 8 suggest that albedo and slope are inversely correlated. The reflectance dip described above is well modeled by a slightly lower albedo on a $8^{\circ}$ slope $4 \mathrm{~km}$ from the starting point, while level areas tend to have higher albedos. Both models resolve two layers, each between 200 and 300 'm thick. The total range of albedo variation in the central section of pro- 

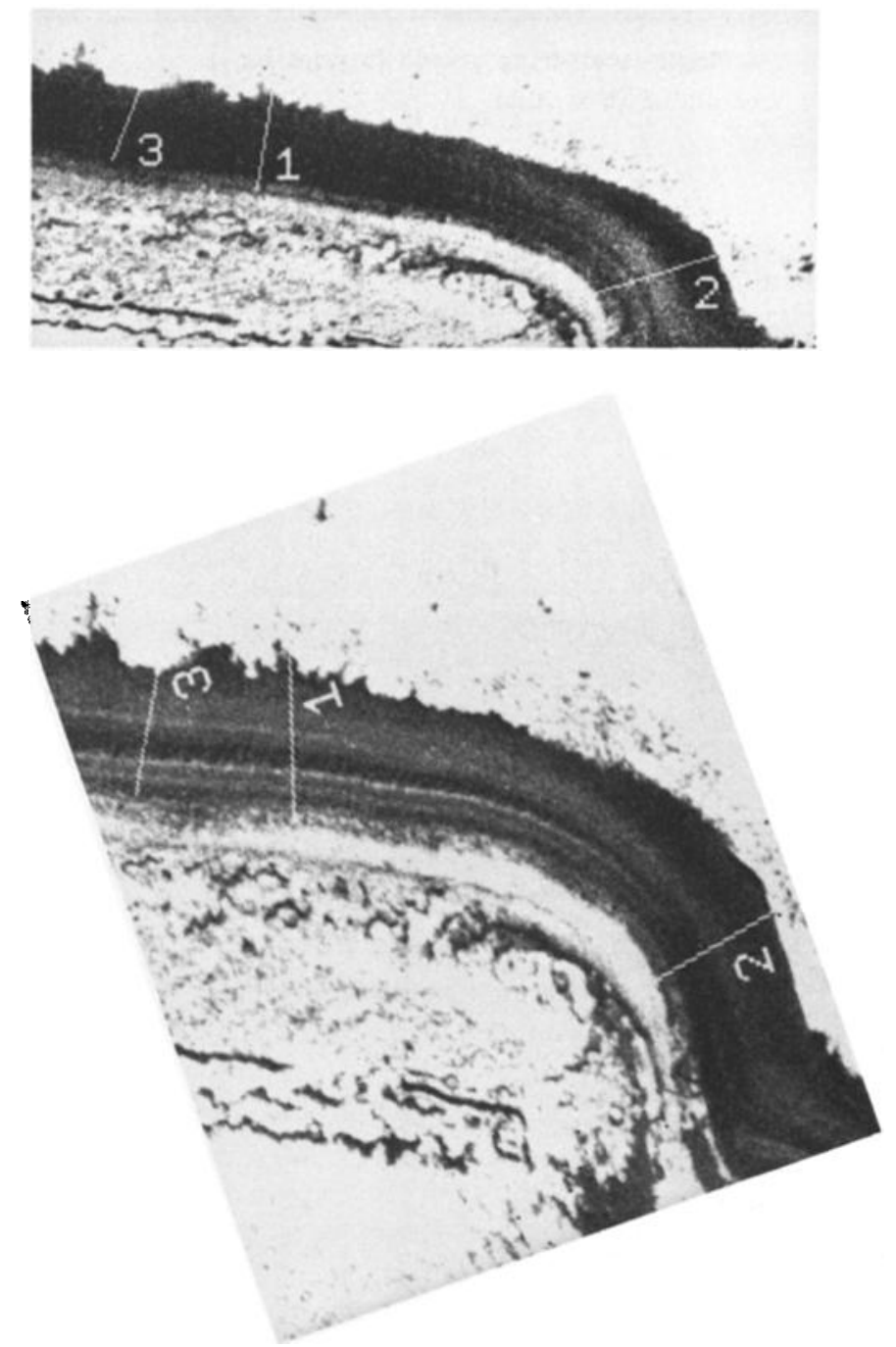

Fig. 9. Portions of images corrected for atmospheric scattering effects, contrast enhanced to display reflectance variations in dark bands: black corresponds to 0.03 , white to 0.16 . Profiles appear to be inclined to layers by different amounts in each image due to oblique viewing. (a) 173B1. (b) 188B2.

file 1 (where not contaminated by the frost cap) is only $12 \%$, less than the noise level in the albedo image. Another profile was therefore analyzed in the same area (Figure 9) to determine if albedo variations are significant, and will be discussed later. First, however, we will introduce the results of our analysis of a profile in a different area.

\section{Profile 2}

Profile 2 is located in a unique, slightly poleward facing exposure of layered deposits (Figure 9). The profile is nearly perpendicular to the solar azimuth in 188B2, so that we shall refer to $188 \mathrm{~B} 2$ as the albedo image in this case. The topography image (173B1) profile (Figure 10a) has the same general shape as that in Figure $4 b$, except that the reflectances 19 pixels from the starting point are much greater than the minimum values around 43 pixels from the start. The albedo profile (Figure 10b) is not as constant as in Figure $4 a$, with a marked increase in reflectance near the middle of the profile. This suggests that the albedo variations in profile $\mathbf{2}$ are much greater than those in profile 1.
As expected, the constant $w$ model does not yield reasonable slopes, as shown in Figure 11. Stereogrammetry indicates only $300 \pm 160 \mathrm{~m}$ of total relief across profile 2 , much less than the total relief in Figure 11 even if the erroneously large slopes (due to the inclusion of polar cap reflectances) near the endpoints are neglected. The reflectance data in the albedo image are not consistent with a constant surface albedo, as illustrated in Figure 12. Because this area does not face equatorward and therefore receives less solar radiation on average than other exposures of layered deposits, the most likely cause of the albedo variations along profile 2 is the presence of frost.

However, even when the surface albedo is allowed to yary along the profile, at least $700 \mathrm{~m}$ of relief is required to model the reflectance data (Figure 13). This amount of relief is definitely outside the limits of uncertainty in the stereogrammetric data, which allow a maximum of $\sim 450 \mathrm{~m}$ of relief on profile 2. The most likely cause of this discrepancy is a temporal change in frost cover between the two images, but we must also consider the effects of foreshortening. 

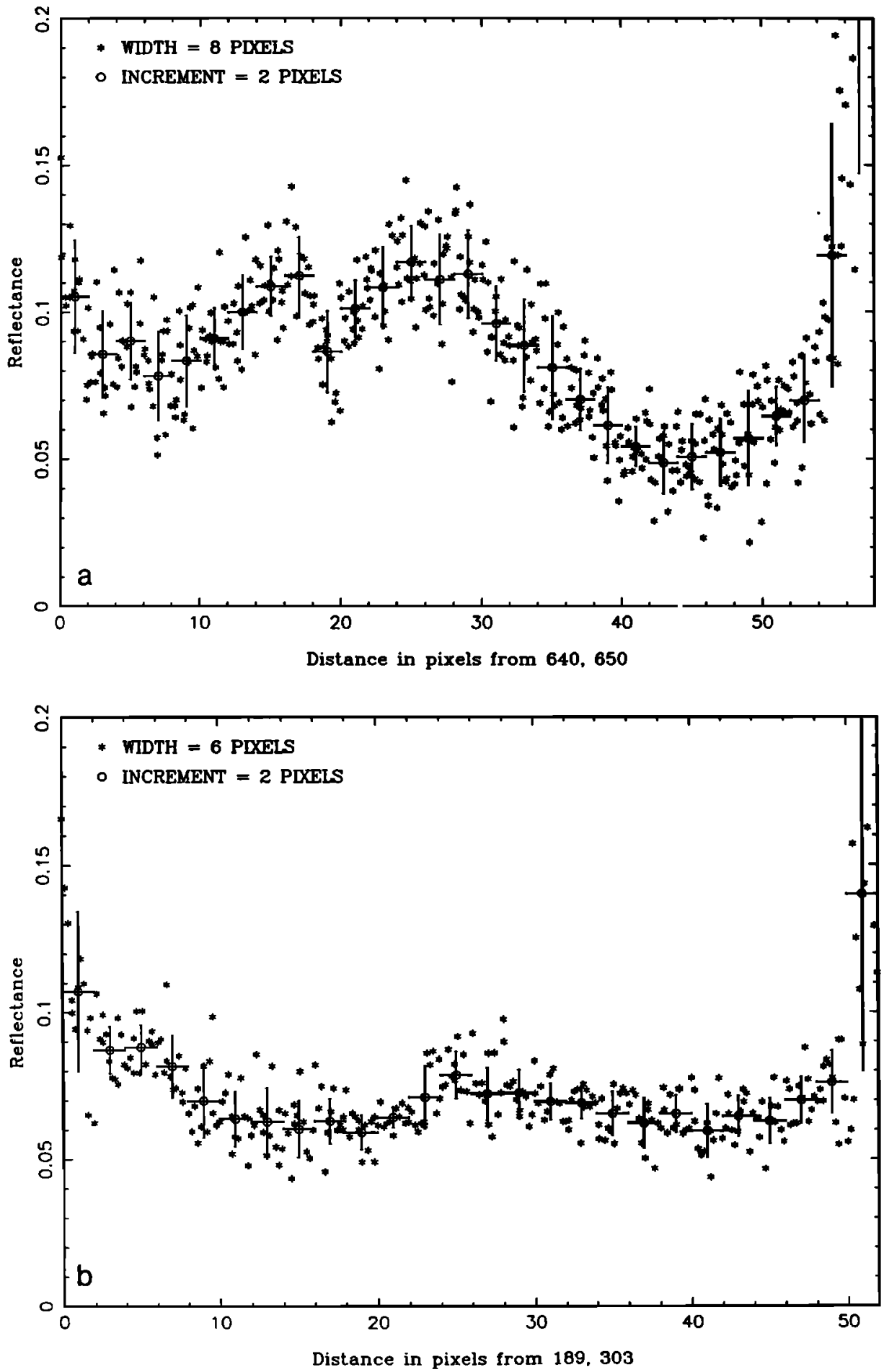

Fig. 10. Profile 2 data with averages of 2-pixel increments. Vertical error bars indicate standard deviation of pixel values in each increment. Increased reflectance near profile endpoints is due to inclusion of residual frost. (a) 173B1 ("topography" image) data from 8-pixel wide swath around profile. (b) $188 B 2$ ("albedo" image) data from 6-pixel wide swath around profile. Note increase in reflectance at center of profile.

Foreshortening, caused by the oblique viewing geometry in these images $\left(\epsilon \approx 46^{\circ}\right)$, has a significant effect on slopes along profile 2 in the albedo image. For example, a $20^{\circ}$ slope will appear $59 \%$ as wide as a level area of the same actual width, if the profile is viewed parallel to its length. Similarly, a $1-\mathrm{km}$ slope will appear only $805 \mathrm{~m}$ wide in $188 \mathrm{B2}$ if its average slope is $10^{\circ}$. Because the resolution of the images used here is no better than $200 \mathrm{~m}$ and the longest continuous slopes are about $1 \mathrm{~km}$ long, foreshortening will have a significant effect only on slopes greater than about $10^{\circ}$ along profile 2 and even less effect on the other profiles. Only "albedo" profiles are viewed nearly parallel to their 


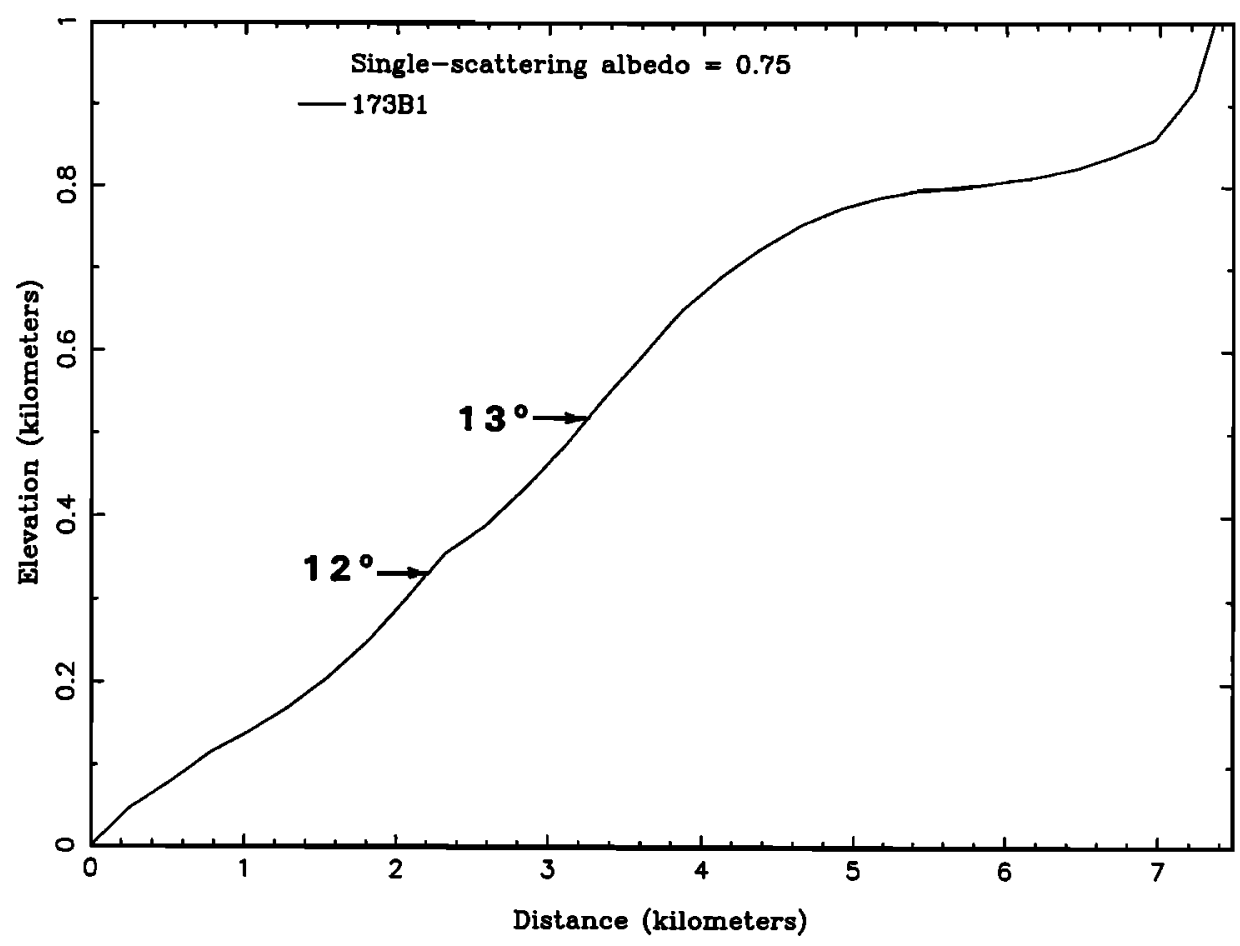

Fig. 11. Constant $w$ model topography for profile 2 with $b=0.5$. Vertical exaggeration $5.4 \times$; maximum slopes indicated.

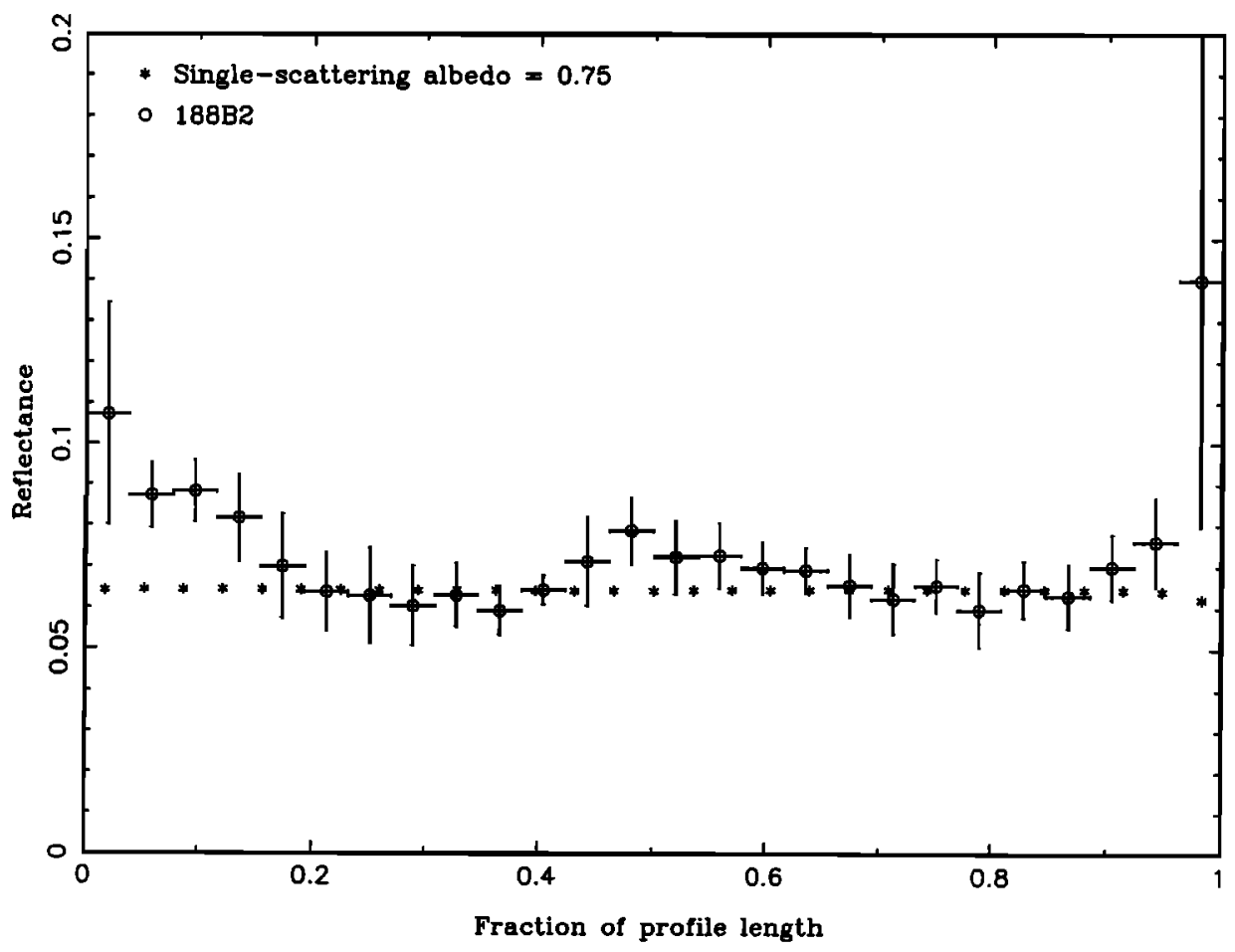

Fig. 12. Comparison of reflectance predicted by model topography with profile 2 data from "albedo" image. Note marked discrepancies between model and data.

length in this study, so that foreshortening has practically no effect on our results. We conclude that foreshortening cannot explain the large overall relief in Figure 13 and that a temporal change in frost coverage along profile 2 is therefore implied.

\section{Profile 3}

The location of profile 3 was chosen to facilitate comparison with the results from profile 1 , as discussed above. In the absence of surface features that could be located in both 


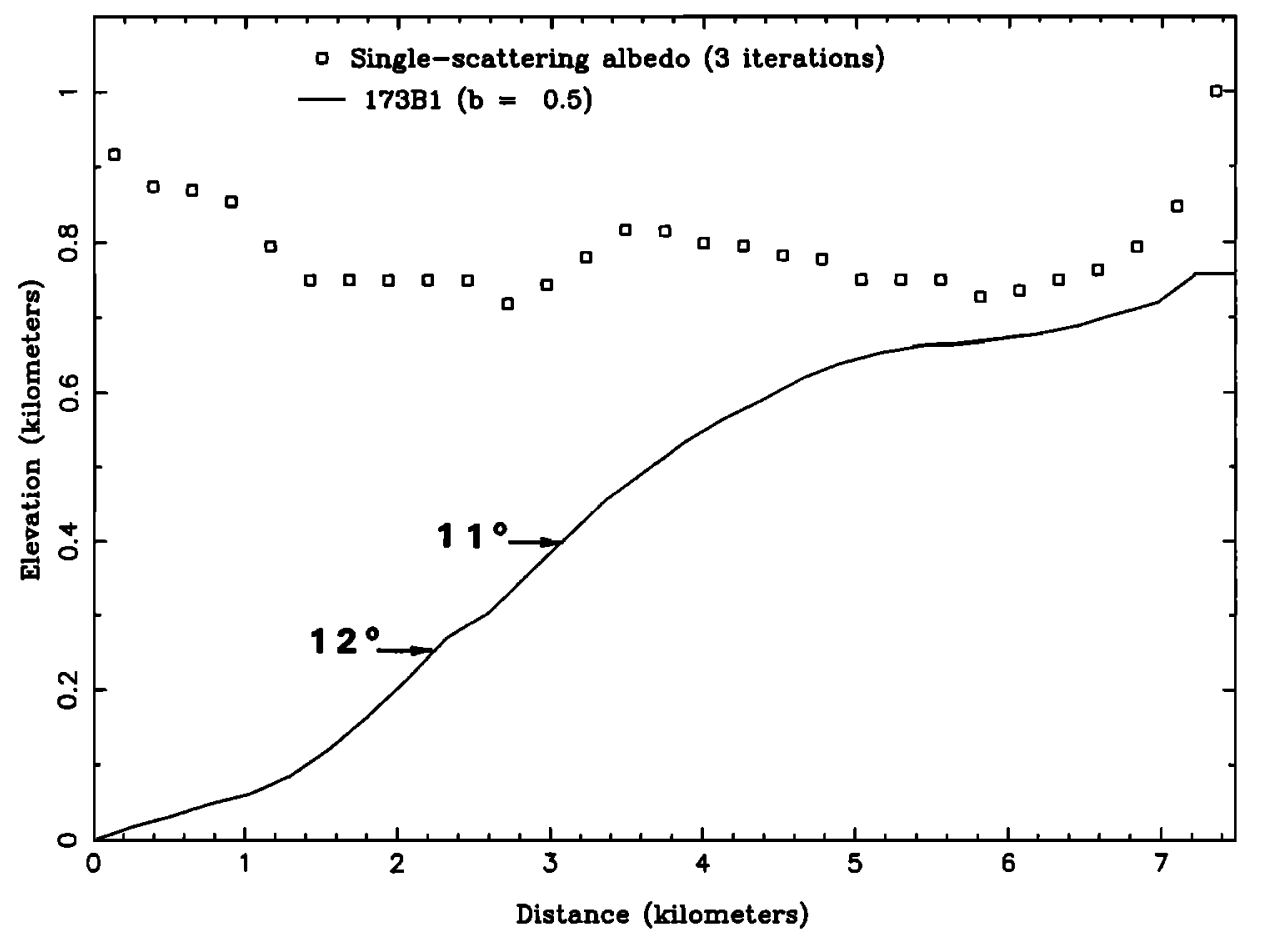

Fig. 13. Profile 2 topography and albedo from iterative model. Elevation and single-scattering albedo are plotted on the same vertical scale. Albedos of 1.0 are not included in the solution. Vertical exaggeration 4.9x; maximum slopes indicated.

images at the base of profile 3 for stereogrammetric measurements, we have approximately located starting points in both images (Figure 9). The starting point in the topography image (188B2) was chosen so that the profile appears perpendicular to the trace of the layers. Because of the oblique viewing geometry, this profile is not actually perpendicular to the strike of the layers. The spacecraft azimuth is nearly perpendicular to the layer strike in the albedo image (173B1), so that the angular relationship between the profile and the layers can be better seen. The endpoint of profile 3 is at the same elevation as the endpoint of profile 1 within $100 \mathrm{~m}$, but the elevation of the start of profile 3 is not precisely known. However, given the stereogrammetric data near the starting points of both profiles (Figure 2), we expect that the total relief across profile 3 will be roughly equal to that across profile $1(500 \mathrm{~m})$.

The endpoints of profile 3 do not extend as far into the polar cap as does profile 1, so that less bright material is included (Figure 14). Profile 3 should therefore be compared to the central section of profile 1 . The two profiles are basically similar in both images, but there are some important differences. The changes in reflectance 20-32 pixels from the start of profile 1 in Figure $4 a$ are not apparent in Figure $14 a$ between 14 and 22 pixels from the start of profile 3 . The reflectance near the end of profile 3 in the topography image (Figure 14b) is never as low as it is 20 pixels from the starting point. The latter difference is due to the irregular boundary of the residual cap: profile 1 extends farther into the cap in a dark embayment (Figure 9). In addition, two bright layers are resolved 7 and 12 pixels from the start of profile 3 (Figure 14b), but are not resolved in Figure $4 b$.

Examination of Figure 9 indicates that the differences between profiles 1 and 3 may be due to lateral variations in the layers, but the images are sufficiently noisy that such a conclusion is debatable in some cases. However, the reflectance dip in the albedo image profile (Figure 14a) 0.4-0.7 from the starting point appears to be due to the presence of a darker layer (Figure 9a). This layer, whether due to topographic or albedo variations, appears to pinch out toward profile 1 , where it is barely visible in the image.

The constant $w$ model was used to produce the topographic profile shown in Figure 15, where the excessive slope at the end of the profile is due to improperly modeled residual frost (as above). The total relief derived is slightly less than that for profile 1 (Figure 6). The reason for the low relief in this case is that the albedo is not constant within the uncertainties in the profile 3 reflectance data, as indicated in Figure 16. The $>1 \sigma$ deviation of all of the model points between 0.5 and 0.7 indicates that the constant $w$ model is not valid in this case.

The general model yields a topographic profile that has the same overall relief as profile 1 (Figure 17), with surface albedo variations of up to $25 \%$. The lower layer in profile 1 is resolved into two 100 -m-thick layers, overlain by a single 300-m-thick layer. The inverse correlation of slope and albedo, suggested by profile 1 results, is much more pronounced in Figure 17. The lowest single-scattering albedo (0.62) occurs on the steepest slope $\left(21^{\circ}\right)$. In addition, two layers near the start of the profile are darkest on their steepest slopes. This relationship suggests that the albedo variations are caused by differential frost retention on exposures of layered deposits, although differences in composition between layers cannot be ruled out entirely.

The inverse correlation of slope and albedo was also noted by Blasius et al. [1982] in the north polar layered deposits. Steep equator-facing slopes defrost more quickly than level 

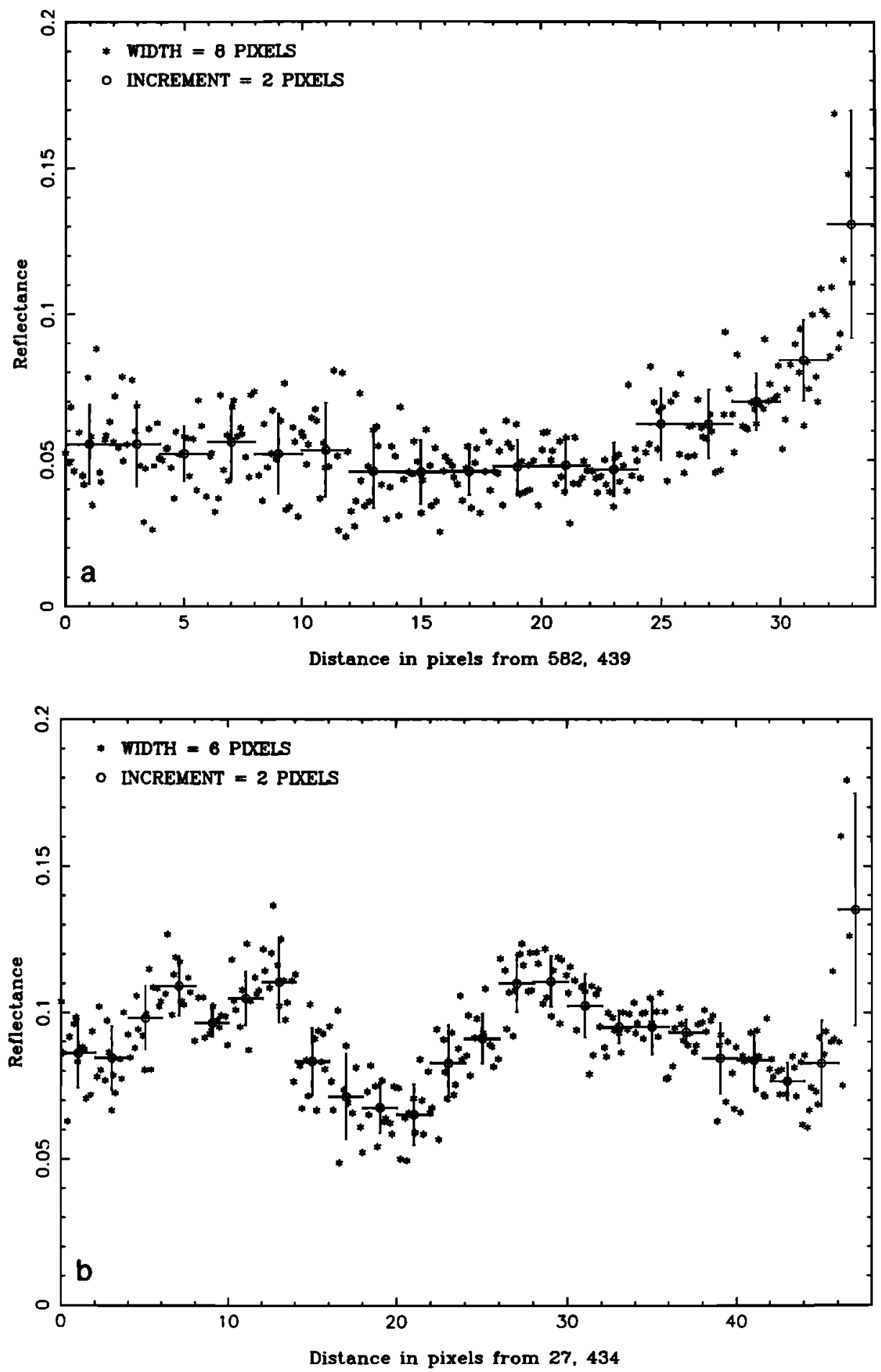

Fig. 14. Profile 3 data with averages of 2-pixel increments. Vertical error bars indicate standard deviation of pixel values in each increment. Increased reflectance near profile endpoints is due to inclusion of residual frost. (a) 173B1 ("albedo" image) data from 8-pixel wide swath around profile. (b) 188B2 ("topography" image) data from 6-pixel wide swath around profile.

areas because of increased insolation. Howard et al. [1982b] found that the frost cover is laterally variable in the northern deposits, as also indicated here by the difference between profiles 1 and 3. Comparison of Figures 8 and 17 shows that more complete defrosting (hence lower albedo) in profile 3 is due to steeper slopes.

The maximum slopes derived here are larger than the $1^{\circ}-8^{\circ}$ slopes reported in the northern layered deposits $[B l a-$ sius et al., 1982]. While the $21^{\circ}$ slope in profile 3 provides the best fit to the data, a slightly smaller slope (and higher albedo) for this layer can also fit the reflectances within their uncertainties. However, as shown in Figure 16, the albedo in this area must be lower than for the rest of the profile. Therefore the slope must be greater than $10^{\circ}$ in order to fit 


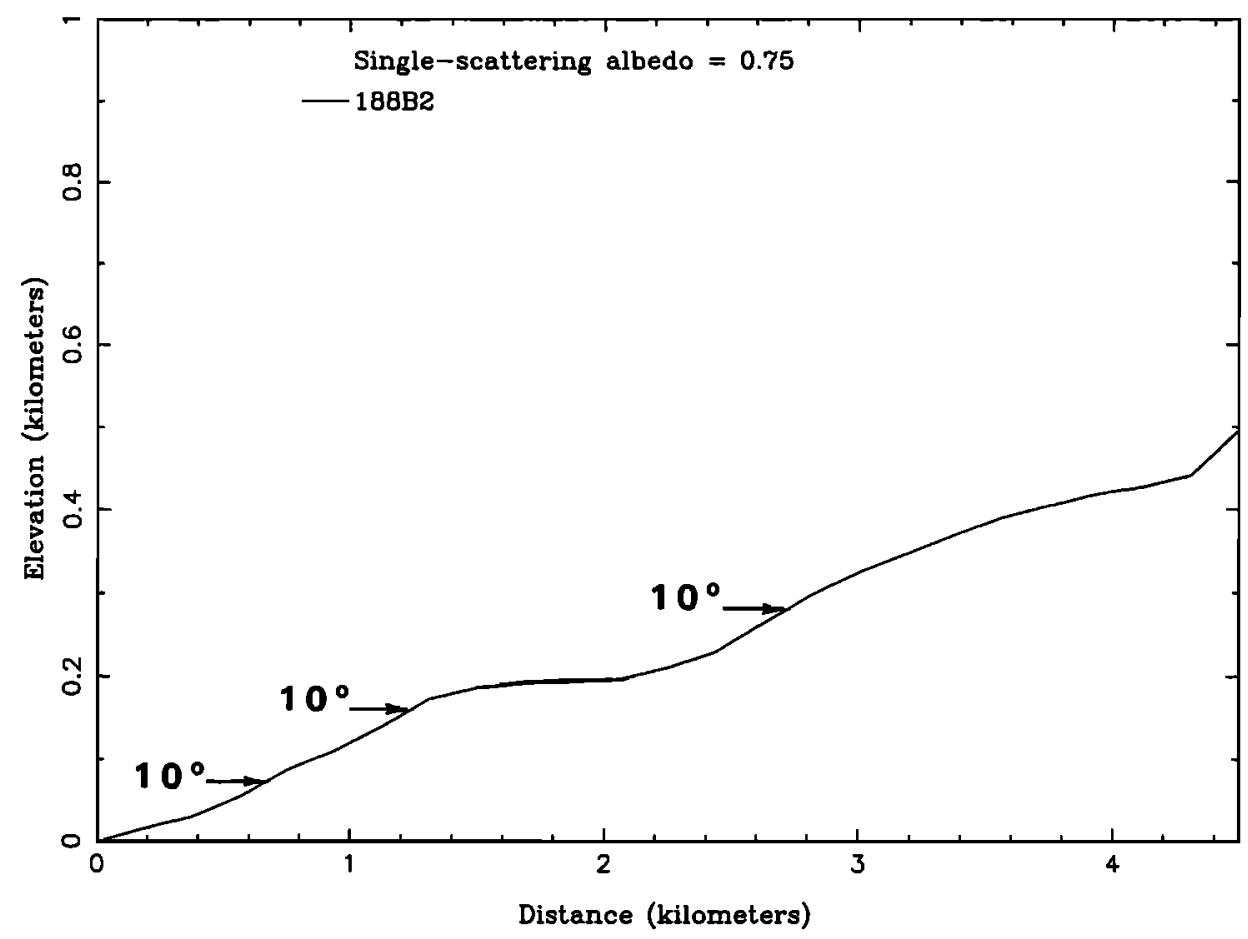

Fig. 15. Constant $w$ model topography for profile 3 with $b=0.5$. Vertical exaggeration $3.25 \times$; maximum slopes indicated. Erroneously large slope at far right due to improper modeling of residual frost.

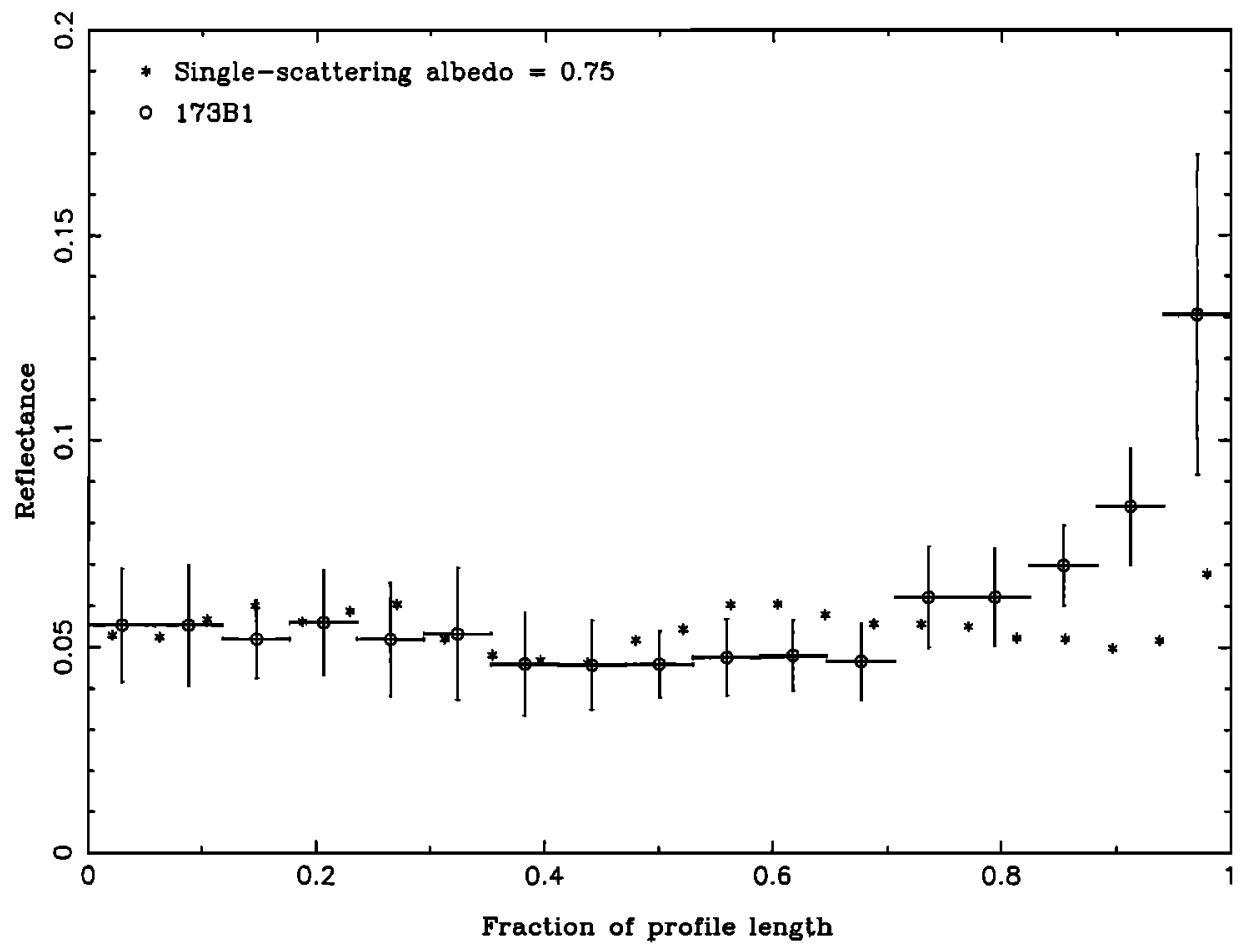

Fig. 16. Comparison of reflectance predicted by model topography with profile 3 data from "albedo" image. Note discrepancies between model and data around 0.6 of profile length.

the reflectance data in both images and is probably at least $15^{\circ}$. The significance of maximum slopes of this magnitude will be discussed after the uncertainties in these results are quantified.

\section{Error Analysis}

The estimated $20 \%$ error in absolute calibration does not directly affect the results reported here. Multiplicative er- rors of this magnitude will not change the ratio of atmospheric to total scattering, and therefore will not significantly change the optical depth fits described above. Because the Lambert albedo used in correcting for atmospheric scattering in $173 \mathrm{~B} 1$ and $188 \mathrm{~B} 2$ was not well constrained by the imaging data, it is not affected by the absolute calibration uncertainty. The single-scattering albedo $w$ and phase function parameter $b$ were also chosen to be consistent with 


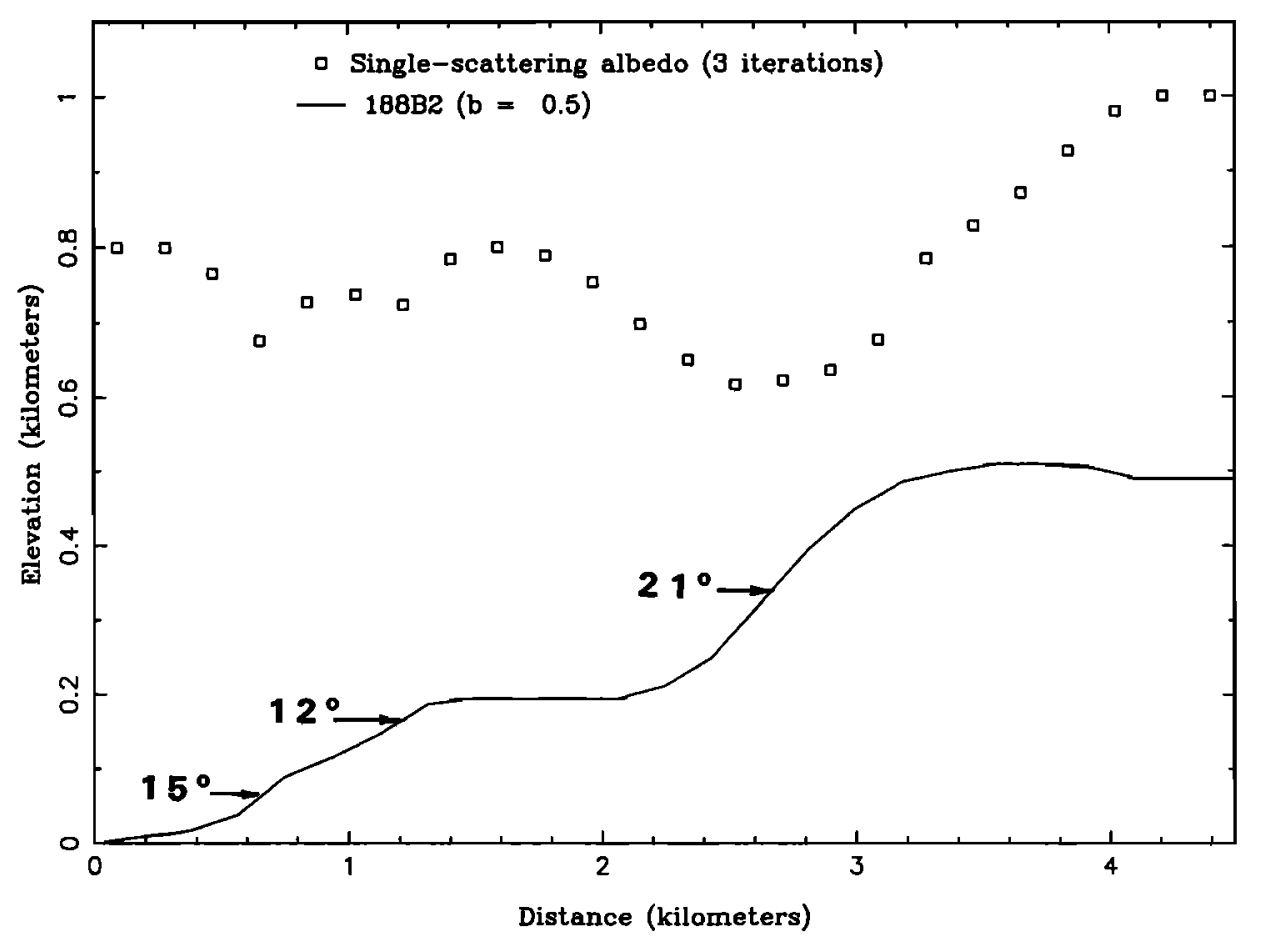

Fig. 17. Profile 3 topography and albedo from iterative model. Elevation and single-scattering albedo are plotted on the same vertical scale. Albedos of $\mathbf{1 . 0}$ are not included in the solution. Note inverse correlation between slope and albedo. Vertical exaggeration $3 \times$; maximum slopes indicated.

stereogrammetric data and are therefore not directly influenced by errors in absolute calibration. However, relative errors are of similar magnitude and have a significant effect on our results.

The noise level in Mariner 9 images is roughly independent of camera response, so that the reflectance uncertainty decreases from $\sim 25 \%$ at 0.05 to $\sim 10 \%$ at 0.10 . These uncertainties are larger than the $8 \%$ relative error predicted by Herkenhoff et al. [1988] because of the low exposure levels in the images (exposed for bright polar cap, not for dark bands). The determination of $w$ is mainly dependent upon the $\sim 25 \%$ uncertainties in the albedo image, corresponding to a $15 \%$ uncertainty in $w$. Hence the $12 \%$ variation in $w$ along profile 1 (Figure 8) is consistent with no variation in $w$ (Figure 6). The uncertainty in the maximum slopes, however, is primarily due to the $\sim 10 \%$ uncertainties in the largest reflectances in the topography image. Reflectance errors of this magnitude result in errors in slope of about $2^{\circ}$. Lower reflectance values in the topography image are more uncertain, so that the shallowest slopes are uncertain by as much as $3^{\circ}$. The slightly negative slopes in Figures 6 and $8 b$ are therefore consistent with a level surface.

Errors in correction for atmospheric effects have a significant effect on the reflectance profiles. In particular, the Lambert surface albedo used in the atmospheric model is rather uncertain, as described above. Varying the Lambert albedo in the atmospheric model results in an additive offset of the reflectance profiles, causing a change in the derived slopes. However (as described above), the Lambert surface albedo was constrained by the stereogrammetric data for profile 1 and therefore does not contribute to the uncertainty in the slopes derived along the other pro- files. Errors in the estimation of the dust optical depth in the atmospheric model cause multiplicative variations in the reflectances that influence the fit of parameters in the photometric function. Once again, however, the agreement between the photoclinometric and stereogrammetric results indicates that these parameters are correct. The uncertainty in correction for atmospheric effects is therefore determined by the uncertainty in the stereogrammetry, so that errors in the atmospheric scattering model do not significantly increase the uncertainty the derived slopes.

Clearly, the error in the overall slope of profile 1 (derived from stereogrammetry) has an effect on the derived slopes. The 90-m relief uncertainty for profile 1 corresponds to an overall slope uncertainty of $\pm 0.7^{\circ}$. This error is small compared to the errors in slope due to reflectance uncertainties, so that the combined error in slope is no more than $3^{\circ}$.

\section{Topography and Albedo of the Layered Deposits}

The photoclinometric profiling presented here confirms the observations, first presented by Murray et al. [1972], that most of the visible layering in the south polar deposits is caused by "staircase" topography. However, the present analysis indicates slopes of about $10^{\circ}-20^{\circ}$ in at least some exposures of layered deposits, steeper than previous estimates of slopes. Terrestrial experience suggests that slopes of this magnitude and length are either composed of large particles or are cemented, although cohesive dust may form $20^{\circ}$ slopes. The largest masses of drift (micron-sized) material around the Viking Landers have slopes of about $20^{\circ}$ [Moore et al., 1987], but these features are at least 2 orders of magnitude smaller than the layered deposit slopes. It is 
difficult to understand how 100-m-high unconsolidated dust slopes could be stable in the presence of strong circumpolar winds for an appreciable period of time. Hence, while the possibility that uncemented dust forms the observed slopes in the southern layered deposits cannot be dismissed entirely, we feel that kilometer- length slopes of loose dust are unlikely. We therefore consider two alternatives for the composition of the layers: uncemented sand-sized particles and cemented dust particles.

Uncemented sand-sized or larger particles can easily form slopes as steep as $20^{\circ}$, but such a composition is at odds with the widely held view that dust is the major non-volatile constituent of the layered deposits [Cutts, 1973; Cutts et al., 1979; Squyres, 1979; Toon et al., 1980; Carr, 1982]. It seems impossible to form laterally extensive layers of almost constant thickness without invoking eolian deposition, so that particles larger than fine sand are ruled out on the grounds that they cannot be plausibly transported by the present Martian atmosphere. The possibility that the layered deposits are composed purely of sand-sized particles is therefore unlikely but cannot be dismissed using the observations presented here.

A more reasonable hypothesis is that the layered deposits contain a significant amount of dust, perhaps with smaller quantities of sand-sized particles [Thomas and Weitz, 1989; Herkenhoff and Murray, 1990]. Some type of cement or cohesion is then needed to stabilize slopes of up to $20^{\circ}$, as discussed above. Water ice is probably not present at the surface of the dark exposures because of the high temperatures expected there during summer [Toon et al., 1980; Hofstadter and Murray, 1990]. Other cementing materials, such as carbonates, cannot be excluded but generally require the presence of liquid water. The brines that may be present at the Viking lander sites [Moore et al., 1987] are generally less stable in the frigid Martian polar regions and are therefore an improbable source of cementing material in the layered deposits. However, we cannot exclude the possibility that dust at the surface of the layered deposits is cemented by salts, as in the case of the blocky material (duricrust) at the Viking lander sites [Moore et al., 1987]. The problem of the instability of fluids that can bring cementing materials to the surface of the polar layered deposits is circumvented by the mechanism proposed below.

The constraints that the layered deposits are composed mainly of dust and that they form $20^{\circ}$ slopes are both satisfied by the formation of a tough weathering rind by erosion of the deposits. Storrs et al. [1988] have shown that sublimation of mixtures of dust and water ice yields a residue of lightweight yet rather sturdy material. Their filamentary sublimation residue (FSR) is composed of particles of various sizes that have been shown by Saunders et al. [1986] to survive extensive saltation. Herkenhoff and Murray [1990] discuss the possibility that FSR particles preferentially form from dark magnetic dust grains and subsequently saltate, forming the dark deposits observed in the south polar region. Such particles may be derived from the weathered surface of the layered deposits, which is evidently competent enough to maintain $20^{\circ}$ slopes. The open structure of the FSR may also serve to insulate underlying water ice from further sublimation. The albedo variations in the layered deposits described above may be due to compositional differences between layers. In this case, variations in source materials and/or atmospheric transport capability may be implied. However, given the temporal changes in albedo between the two images studied here, we conclude that variations in frost cover are the cause of the albedo differences. This interpretation is supported by the increased albedo observed along profile 2, which slopes toward the south pole. The $25 \%$ variation in albedo along profile 3 exceeds the $15 \%$ uncertainty in albedo determination, indicating that our model results are robust. The minimum (unfrosted) surface singlescattering albedo of $0.62 \pm 0.09$ at $0.56 \mu \mathrm{m}$ is consistent with values of $w$ found by Thorpe [1982] and Arvidson et al. [1989] in Arabia and Chryse.

If the albedo variations between layers are due mainly to differences in frost coverage, the intrinsic variations in albedo between resolved layers are constrained to be less than $15 \%$. This suggests that compositional variations in the non-volatile component of the deposits are minor. However, thin layers with different compositions and albedos may be present and simply not resolved in the images used here.

\section{SUMMARY AND CONCLUSIONS}

Photoclinometric analysis of exposures of the south polar layered deposits indicates that slopes of up to $21^{\circ} \pm 2^{\circ}$ occur locally. There is evidence that the slopes within layered deposit exposures vary laterally. Frost is preferentially retained on level areas late into the summer and sublimed from steep equator-facing slopes, causing surface albedo variations of up to $25 \pm 15 \%$. Interlayer variations in albedo are constrained to be less than $15 \%$, implying that the differences in resistance to erosion are caused by variations in ice content unless unresolved layers of different (nonvolatile) composition are present. The minimum single-scattering albedo is similar to that observed in dusty areas on Mars, suggesting that dust is a major component of the layered deposits. Layer thickmesses of 100-300 $\mathrm{m}$ are observed, but thinner (unresolved) layers are possible or even probable.

The steepness of the slopes and consideration of the instability of water ice at the surface of the layered deposits suggests that slopes on layered deposit exposures may be maintained by the presence of a strong weathering rind. In the absence of such a competent surface layer, sublimation of water ice from the layered deposits would leave only loose dust (and perhaps some sand-sized particles) that would presumably slump down or blow away. In either case, the removal of dust would expose more water ice to the sun, resulting in rapid erosion of the layered deposits. Calculations by Toon et al. [1980] show that about $15 \mathrm{~cm} \mathrm{yr}^{-1}$ of water ice would be sublimed from the layered deposits at $80^{\circ} \mathrm{S}$ latitude if the $\mathrm{H}_{2} \mathrm{O}$ were not protected from the sun. Even if the layered deposits are mostly ice, this indicates that a few vertical kilometers per million years of the deposits could be removed by this type of erosion. Sunward-facing scarps would be expected to retreat at a much higher rate. This result suggests that water ice in the layered deposits is protected by a surface layer of some kind that insulates the $\mathrm{H}_{2} \mathrm{O}$ from solar heating [Hofstadter and Murray, 1990]. We propose that this surface layer is a weathering rind composed of dark, self-cementing sublimation residue particles, such as those created in the experiments described by Saunders et al. [1986]. This hypothesis is consistent with the regional 
color and albedo of the southern layered deposits, which indicates that layered deposit "bedrock" is slightly darker and less red than the bright dust that mantles the surface [Herkenhoff and Murray, 1990].

The results presented in this paper, along with previous studies, suggest the following scenario for layered deposit evolution. Dark, magnetic dust motes preferentially form larger (sand-sized) residue particles upon sublimation of water ice from the layered deposits [Herkenhoff and Murray, 1990]. Weathering of the deposits produces a strong surface layer of such particles that effectively insulates water ice deeper within the layered deposits and permits $10^{\circ}-20^{\circ}$ slopes to be stable. Further erosion loosens the dark residue particles, allowing them to be transported by saltation and build the dunes observed in both the north and south polar regions.

If this hypothesis is correct, the exposures studied here should be somewhat darker than the bright dust that mantles much of the south polar layered deposits. The dark band in Figure 9 is not well resolved in the Viking orbiter 2 color mosaic studied by Herkenhoff [1989], so that the regional color and albedo of this exposure is not easily quantified. Further study is therefore required to adequately test this hypothesis.

The stepped topography on layered deposit outcrops is probably caused by variations in resistance to erosion between layers [Howard, 1978]. Such variations in erosive resistance may be due to differences in susceptibility to sublimation of water ice. Differences in ice/dust content and in dust composition may cause such variations in erosion rate. However, variations in dust composition must not result in surface albedo changes greater than $15 \%$. The magnitude of surface slopes provides constraints on possible mechanisms for layered deposit erosion.

Future studies of the topography and albedo of the layered deposits should include examination of areas far from the residual cap, where there is less surface frost. Current investigations of the south polar deposits using the technique described here are limited by the availability and quality of Mariner 9 stereo coverage. The Mars observer camera, as currently planned, should be able to obtain images of the layered deposits that are well suited to analysis by this technique. In particular, it may be possible to image key areas near the poles at different times of day during the summer, providing the different solar illuminations required. Such high-resolution images will probably resolve thinner layers and further constrain hypotheses for the origin and evolution of the deposits. In addition, the laser altimeter on Mars observer will hopefully provide regional topographic data in the polar regions and better height control than the stereogrammetry used in this study.

Acknowledgments. We thank Phil Davis, Kay Edwards, Randy Kirk, Alfred McEwen, and Larry Soderblom for helpful suggestions and assistance and Yuk Yung for allowing us to use his RADIATE program to model the Martian atmosphere. Reviews by two anonymous referees are also gratefully acknowledged. This work was supported by NASA grant NAGW-1226. Contribution number $\mathbf{4 7 7 2}$ of the Division of Geological and Planetary Sciences.

\section{REFERENCES}

Arvidson, R. E., E. A. Guinness, M. A. Dale-Bannister, J. Adams, M. Smith, P. R. Christensen, and R. B. Singer, Nature and distribution of surficial deposits in Chryse Planitia and vicinity, Mars, J. Geophys. Res., 94, 1573-1587, 1989.
Batson, R. M., Digital cartography of the planets: New methods, its status, and its future, Photogramm. Eng. Remote Sens., 5S, 1211-1218, 1987.

Blasius, K. R., A study of Martian topography by analytic photogrammetry, J. Geophys. Res., 78, 4411-4423, 1973.

Blasius, K. R., J. A. Cutts, and A. D. Howard, Topography and stratigraphy of Martian polar layered deposits, Icarus, 50, 140$160,1982$.

Buratti, B. J. and J. Veverka, Photometry of rough planetary surfaces: The role of multiple scattering, Icarus, 64, 320-328, 1985.

Carr, M. H., Periodic climate change on Mars: Review of evidence and effects on distribution of volatiles, Icarus, 50, 129-139, 1982.

Cutts, J. A., Nature and origin of layered deposits of the Martian polar regions, J. Geophys. Res., 78, 4231-4249, 1973.

Cutts, J. A., Mariner Mars 1971 television picture catalog: Experiment design and picture data, Tech. Memo. 39-585, vol. 1, Jet Propul. Lab., Pasadena, Calif., 1974.

Cutts, J. A., K. R. Blasius, and W. J. Roberts, Evolution of Martian polar landscapes: Interplay of long-term variations in perennial ice cover and dust storm intensity, J. Geophys. Res., 84, 2975-2994, 1979.

Davis, P. A. and L. A. Soderblom, Modeling crater topography and albedo from the monoscopic Viking orbiter images, 1, Methodology, J. Geophys. Res., 89, 9449-9457, 1984.

Dzurisin, D. and K. R. Blasius, Topography of the polar layered deposits of Mars, J. Geophys. Res., 80, 3286-3306, 1975.

Edwards, K., Geometric processing of digital images of the planets, Photogramm. Eng. Remote Sens., 58, 1219-1222, 1987.

Hapke, B., Bidirectional reflectance spectroscopy, 1, Theory, $J$. Geophys. Res., 86, 3039-3054, 1981.

Hapke, B., Bidirectional reflectance spectroscopy, 3, Correction for macroscopic roughness, Icarus, 59, 41-59, 1984.

Helfenstein, P., The geological interpretation of photometric surface roughness, Icarus, 78, 462-481, 1988.

Herkenhoff, K. E., Quantitative studies of the Martian south polar region using spacecraft images, $\mathrm{Ph}$.D. thesis, Calif. Inst. of Technol., Pasadena, 1989.

Herkenhoff, K. E. and B. C. Murray, Color and albedo of the south polar layered deposits on Mars, J. Geophys. Res., 95, 1343-1358, 1990.

Herkenhoff, K. E., L. A. Soderblom, B. C. Murray, and G. E. Danielson, Mariner 9 television calibration-Revisited, Icarus, 75, 133-145, 1988.

Hofstadter, M. D. and B. C. Murray, Ice sublimation and rheology: Implications for the Martian polar layered deposits, Icarus, 84, 352-361, 1990.

Howard, A. D., Origin of the stepped topography of the Martian poles, Icarres, S4, 581-599, 1978.

Howard, A. D., K. R. Blasius, and J. A. Cutts, Photoclinometric determination of the topography of the Martian north polar cap, Icares, 50, 245-258, 1982 .

Howard, A. D., J. A. Cutts, and K. R. Blasius, Stratigraphic relationships within Martian polar cap deposits, Icarus, 50, 161215, $1982 b$.

Klaasen, K. P., T. E. Thorpe, and L. A. Morabito, Inflight performance of the Viking visual imaging subsystem, Applied Optics, 16, 3158-3170, 1977.

McEwen, A. S., Topography and albedo of Ius Chasma, Mars, Lunar Planet. Sci., XVI, 528-529, 1985.

Moore, H. J., R. E. Hutton, G. D. Clow, and C. R. Spitzer, Physical properties of the surface materials at the Viking landing sites on Mars, U.S. Geol. Surv. Prof. Pap., 1989, 222 pp., 1987.

Murray, B. C., L. A. Soderblom, J. A. Cutts, R. P. Sharp, D. J. Milton, and R. B. Leighton, Geological framework of the south polar region of Mars, Icarus, 17, 328-345, 1972.

Pang, K. D. and C. W. Hord, Mariner 9 ultraviolet spectrometer experiment: 1971 Mars dust storm, Icarus, 18, 481-488, 1973.

Saunders, R. S., F. P. Fanale, T. J. Parker, J. B. Stephens, and S. Sutton, Properties of filamentary sublimation residues from dispersions of clay in ice, Icarus, 66, 94-104, 1986.

Squyres, S. W., The evolution of dust deposits in the Martian north polar region, Icarus, 40, 244-261, 1979.

Storrs, A. D., F. P. Fanale, R. S. Saunders, and J. B. Stephens, The formation of filamentary sublimate residues (FSR) from mineral grains, Icarus, $76,493-512,1988$. 
Thomas, P., K. Herkenhoff, A. Howard, B. Murray, and S. Squyres, Polar deposits on Mars, in Mors. University of Arizona Preas, Tucson, in press, 1990.

Thomas, P. C. and C. Weitz, Sand dune materials and polar layered depositg on Mars. Icarus, 81, 185-215, 1989.

Thorpe, T. E., Verification of performance of the Mariner 9 television cameras, Appl. Opt., 12, 1775-1784, 1973.

Thorpe, T. E., Martian surface properties indicated by the opposition effect, Icarus, 49, 398-415, 1982.

Toon, O. B., J. B. Pollack, W. Ward, J. A. Burns, and K. Bilski, The astronomical theory of climatic change on Mars. Icarus, 14 , 552-607, 1980.

Veverka, J., P. Thomas, T. V. Johnson, D. L. Matson, and K.
Housen, The physical characteristics of satellite surfaces, in Satellites, edited by J. Burns and M. S. Matthews, pp. 342-402, Univervity of Arizona Press, Tucson, 1986.

K. E. Herkenhoff, Jet Propulaion Laboratory 183-501, 4800 Oak Grove Drive, Pasadena, CA 91109.

B. C. Murray, Division of Geological and Planetary Sciences, California Institute of Technology, Pasadena, CA 91125.

(Received July 5, 1989;

(revised December 11, 1989;

accepted December 13, 1989.) 